\title{
On the Theory of Wave Propagation Through a Concentrically Stratified Troposphere With a Smooth Profile. Part II. Expansion of the Rigorous Solution
}

\section{H. Bremmer}

Contribution from Philips Research Laboratories, N. V. Philips Gloeilampenfabrieken, Eindhoven, Netherlands

(Received May 15, 1961 ; revised July 10, 1961)

\begin{abstract}
This part concerns the height-gain differential equation in order to obtain a series for the complete solution which starts with the extended W. K. B. approximation discussed in part I. The coefficients of this equation depend for each mode amongst other things on the parameters $\Lambda_{j}$ fixing the refractive-index profile. However, the explicit dependence on these parameters can only be given in terms of expansions with respect to $\left(k_{0} a\right)^{-2 / 3}\left(k_{0} a=\right.$ circumference of the earth divided by the wavelength). In turn these expansions are derived with the aid of other ones for the complex turning point connected with the height-gain differential equation. The final expansion for the solution of the differential equation is substituted in the boundary condition at the earth's surface. This leads to corresponding expansions, with respect to $\left(k_{0} a\right)^{-2 / 3}$, of the quantity $u_{l}(a)$, and next of the eigenvalues $l$ themselves.
\end{abstract}

\section{Canonical Form of the Height-Gain Differential Equation}

According to section 3 the field of a dipole situated in a spherically symmetric stratified medium can be expressed in terms of a single scalar quantity $\Pi$. The latter can be expanded in modes $f_{l}(r) P_{l}(\cos \theta)$ the radial parts of which have to satisfy the height-gain differential eq (8), viz,

$$
\left\{\frac{d^{2}}{d r^{2}}+k_{0}^{2} m_{l}^{2}(r)\right\}\left\{r f_{l}(r)\right\}=0 .
$$

According to (9), (14), and (15) $m_{l}$ is defined as follows in terms of the profile $M_{\text {eff }}=(r / a) n_{\text {eff }}$ :

$$
m_{l}(r)=\frac{a}{r} \sqrt{M_{\mathrm{eff}}^{2}(r)-C_{l}^{2}}
$$

Moreover, the height-gain functions should fulfil the radiation condition at infinity, as well as the boundary condition (23) at the earth's surface.

Following the methods of Langer [1937] and Pekeris [1946] we introduce the new variables:

$$
u_{l}(r)=k_{0} \int_{r_{l}}^{r} m_{l}(s) d s=k_{0} a \int_{r_{l}}^{r} \frac{d s}{s} \sqrt{M_{\text {eff }}^{2}(s)-C_{l}^{2}}
$$

and

$$
G_{l}=\frac{r}{a} \sqrt{m_{l}(r)} f_{l}(r)=\sqrt{\frac{r}{a}}\left\{M_{\text {eff }}^{2}(r)-C_{l}^{2}\right\}^{1 / 4} f_{l}(r),
$$

the former of which has been discussed in section 5. The influence of the refractive-index profile enters explicitly by the occurrence of $M_{\text {eff }}$, and implicitly by that of the turning-point level $r_{l}$ defined by (14). The height-gain differential equation then proves to be equivalent to the new equation:

$$
\frac{d^{2} G_{l}}{d u_{l}^{2}}+P_{l}\left(u_{l}\right) G_{l}=0
$$

\footnotetext{
1 Part I. J. Research NBS 64D (Radio Prop.) No. 5, 467-482 (Sept.-Oct. 1960).
} 
in which

$$
P_{l}\left(u_{l}\right) \equiv 1+\frac{1}{4} \frac{\left(\frac{d m_{l}}{d u_{l}}\right)^{2}}{m_{l}^{2}}-\frac{1}{2} \frac{\frac{d^{2} m_{l}}{d u_{l}^{2}}}{m_{l}} .
$$

The eq(68) will be the starting point for our further investigations.

\section{First Expansion for $m_{l}(r)$ as a Function of $u_{l}(r)$, With Coefficients Depending on $r_{l}$}

In order to solve (68) we have to determine $P_{l}\left(u_{l}\right)$ as a function of $u_{l}$. This can be done for each eigenvalue $l$ in terms of an expansion the coefficients of which depend on the parameters $\Lambda_{\text {f }}$ of (17). The latter characterize the refractive-index profile. In view of (69) we start with the determination of $m_{l}$ as a function of $u_{l}$. The tedious derivation of the coefficients of the corresponding expansion will be given in steps, to be discussed in the next sections.

We first notice that $m_{l}^{2}$ could be expanded into a Taylor series in $r-r_{l}$, since $m_{l}^{2}$ is a continuous function of $r$ according to (65). However, this series starts with the linear term; in fact, the relation $M_{\text {eff }}\left(r_{l}\right)=C_{l}$ (see (14)) involves $m_{l}\left(r_{l}\right)=0$. We infer a proportionality of $m_{l}(r)$ with $\left(r-r_{l}\right)^{\frac{1}{2}}$ for small values of $r-r_{l}$. In view of (66) this involves a corresponding proportionality of $u_{l}$ with $\left(r-r_{l}\right)^{3 / 2}$, or of $r-r_{l}$ with $u_{l}^{2 / 3}$, and therefore of $m_{l}$ with $u_{l}^{1 / 3}$. Similar considerations show the proportionality of the next terms of the expansion of $m_{l}$ (in terms of $u_{l}$ ) with $u_{l}^{3 / 3}$, $u_{l}^{5 / 3}, u_{l}^{7 / 3}$, etc. We therefore put:

$$
\frac{d u_{l}}{d r}=k_{0} m_{l}=\frac{1}{a} \sum_{n=0}^{\infty} B_{n}^{(l)} \frac{u_{l}^{\frac{1}{3}(2 n+1)}}{\left(k_{0} a\right)^{\frac{2}{3}(n-1)}},
$$

the dimensionless coefficients $B_{n}^{(l)}$ of which are to be determined next. In this section these coefficients will be connected with the properties of the profile near the turning point $r_{l}$.

We first multiply the expansion (70) by itself, so as to obtain

$$
k_{0}^{2} a^{2} m_{l}^{2}=\sum_{j=0}^{\infty} \sum_{s=0}^{\infty} B_{j}^{(l)} B_{s}^{(l)} \frac{u_{l}^{\frac{2}{3}(j+s+1)}}{\left(k_{0} a\right)^{\frac{2}{3}(j+s-2)}} .
$$

We substitute $s=m-j$, and invert the order of summation with respect to $j$ and $m$. The resulting expansion reads:

$$
k_{0}^{2} a^{2} m_{l}^{2}=\sum_{m=0}^{\infty} \frac{u_{l}^{\frac{2}{3}(m+1)}}{\left(k_{0} a\right)^{\frac{2}{3}(m-2)}} \sum_{j=0}^{m} B_{j}^{(l)} B_{m-j}^{(l)} \cdot
$$

The coefficients $B_{j}^{(l)}$ can now be obtained from the derivatives of (71) with respect to $r$, taking $r=r_{l}$. For instance, the first order derivative yields, applying both (70) and (71),

$$
\begin{aligned}
k_{0}^{2} a^{3} \frac{d\left(m_{l}^{2}\right)}{d r}=\frac{d}{d u_{l}} & \left\{\sum_{m=0}^{\infty} \frac{u_{l}^{\frac{2}{3}(m+1)}}{\left(k_{0} a\right)^{\frac{2}{3}(m-2)}} \sum_{j=0}^{m} B_{j}^{(l)} B_{m-j}^{(l)}\right\} \\
& \times \sum_{n=0}^{\infty} B_{n}^{(l)} \frac{u_{l^{\frac{1}{3}}(2 n+1)}}{\left(k_{0} a\right)^{\frac{2}{3}(n-1)}} \cdot
\end{aligned}
$$

Since $r=r_{l}$ involves $u_{l}=0$, we have only to derive the term $m=n=0$ in order to get the value of the left-hand side at $r=r_{l}$. Similarly, a further differentiation of (72) with respect to $u$, multiplied by (70), yields the second derivative of $m_{l}^{2}$; its value at $r=r_{l}$ reduces to the constant term of this new expansion, and so on.

We mention the expressions thus obtained for the first few derivatives of $m_{l}^{2}$ at $r=r_{l}$ :

$$
\begin{gathered}
\eta_{l}^{\prime} \equiv a\left\{\frac{d\left(m_{l}^{2}\right)}{d r}\right\}_{r=r_{l}}=\frac{2}{3}\left\{B_{0}^{(l)}\right\}^{3} ; \\
\eta_{l}^{\prime \prime} \equiv a^{2}\left\{\frac{d^{2}\left(m_{l}^{2}\right)}{d r^{2}}\right\}_{r=r_{l}}=\frac{20}{9} B_{0}^{(l) 3} B_{1}^{(l)} ;
\end{gathered}
$$




$$
\begin{gathered}
\eta_{l}^{\prime \prime \prime} \equiv a^{3}\left\{\frac{d^{3}\left(m_{l}^{2}\right)}{d r^{3}}\right\}_{r=r_{l}}=\frac{8}{27} B_{0}^{(l) 3}\left\{14 B_{0}^{(l)} B_{2}^{(l)}+19 B_{1}^{(l) 2}\right\} ; \\
\eta_{l}^{I V} \equiv a^{4}\left\{\frac{d^{4}\left(m_{l}^{2}\right)}{d r^{4}}\right\}_{r=r_{l}}=\frac{16}{81}\left\{B_{0}^{(l)}\right\}^{3}\left\{160 B_{0}^{(l)} B_{1}^{(l)} B_{2}^{(l)}+54 B_{0}^{(l)}{ }^{2} B_{3}^{(l)}+65 B_{1}^{(l)^{3}}\right\}
\end{gathered}
$$

From these relations we may solve in succession the coefficients $B_{o}^{(l)}, B_{1}^{(l)}$, etc. We obtain:

$$
\begin{aligned}
B_{0}^{(l)} & =\left\{\frac{3 \eta_{l}^{\prime}}{2}\right\}^{1 / 3} ; \\
B_{1}^{(l)} & =\frac{3}{10}\left\{\frac{\eta_{l}^{\prime \prime}}{\eta_{l}^{\prime}}\right\}^{\prime} ; \\
B_{2}^{(l)} & =\frac{9}{56}\left\{\frac{2}{3 \eta_{l}^{\prime}}\right\}^{1 / 3}\left\{\frac{\eta_{l}^{\prime \prime \prime}}{\eta_{l}^{\prime}}-\frac{19}{25} \frac{\eta_{l}^{\prime \prime 2}}{\eta_{l}^{\prime 2}}\right\} ; \\
B_{3}^{(l)} & =\left\{\frac{2}{3 \eta_{l}^{\prime}}\right\}^{2 / 3}\left\{\frac{\eta_{l}^{I V}}{16 \eta_{l}^{\prime}}-\frac{\eta_{l}^{\prime \prime} \eta_{l}^{\prime \prime \prime}}{7 \eta_{l}^{\prime 2}}+\frac{213}{2800} \frac{\eta_{l}^{\prime \prime 3}}{\eta_{l}^{\prime 3}}\right\}
\end{aligned}
$$

\section{Second Expansion for $m_{l}(r)$ as a Function of $u_{l}(r)$ With Coefficents Depending on the Profile-Function $M(r)$}

According to (73) and (74) the coefficients $B_{n}^{(l)}$ of (70) depend on the position of the turning point $r_{l}$ which may be derived from (18). In this latter expansion the parameters $\Lambda_{j}$ are parameters known from the given profile of the refractive index. In order to obtain the turning point from (18) in its complete explicit dependence from the profile, we shall have to know the coefficients $m_{l}(a)$ entering in (18). Therefore, we next want to obtain a connection between the latter and the profile parameters $\Lambda_{j}$. It can be arrived at from an expansion of $m_{l}(r)$ in terms of $u_{l}(r)$, to be derived as follows.

We start from the expansion (21). In view of (65) it can be represented by

in which

$$
u_{l}(r)=k_{0} a \sum_{j=0}^{\infty} \alpha_{j}(r) m_{l}(r)^{2 j+3}
$$

$$
\alpha_{j}(r) \equiv(-1)^{j} \frac{2}{3} \frac{2}{5} \frac{2}{7} \cdots \frac{2}{2 j+3} \frac{d^{j}}{d\left\{\boldsymbol{M}_{\mathrm{eff}}^{2}(r)\right\}^{j}}\left\{\frac{1}{r \frac{d \boldsymbol{M}_{\mathrm{eff}}^{2}}{d r}}\right\} .
$$

We invert this series, taking into account that the first term proportional to $m_{l}^{3}$ in the original series involves a first term proportional to $u_{l}^{1 / 3}$ in the new one. The complete inversion series can be put in the form:

$$
m_{l}(r)=C_{0}(r)\left\{\frac{u_{l}(r)}{k_{0} a}\right\}^{1 / 3}+C_{1}(r)\left\{\frac{u_{l}(r)}{k_{0} a}\right\}^{3 / 3}+C_{2}(r)\left\{\frac{u_{l}(r)}{k_{0} a}\right\}^{5 / 3}+C_{3}(r)\left\{\frac{u_{l}(r)}{k_{0} a}\right\}^{7 / 3}+\ldots
$$

In fact, substituting this expansion itself and the corresponding expansions for its successive powers, such as

$$
m_{l}^{2}(r)=C_{0}^{2}\left\{\frac{u_{l}}{k_{0} a}\right\}^{2 / 3}+2 C_{0} C_{1}\left\{\frac{u_{l}}{k_{0} a}\right\}^{4 / 3}+\left(C_{1}^{2}+2 C_{0} C_{2}\right)\left\{\frac{u_{l}}{k_{0} a}\right\}^{6 / 3}+2\left(C_{0} C_{3}+C_{1} C_{2}\right)\left\{\frac{u_{l}}{k_{0} a}\right\}^{8 / 3} \ldots
$$

into (75), we find by equating the corresponding powers of $u_{l}$ in both members unique relations for the determination of the new coefficients $C_{j}(r)$ from the other coefficients $\alpha_{j}(r)$. The first relations of this type read explicitly: 


$$
\begin{aligned}
& C_{0}(r)=\frac{1}{\left\{\alpha_{0}(r)\right\}^{1 / 3}}, \\
& C_{1}(r)=\frac{-\alpha_{1}(r)}{3 \alpha_{0}^{2}(r)}, \\
& C_{2}(r)=\frac{4 \alpha_{1}^{2}(r)-3 \alpha_{0}(r) \alpha_{2}(r)}{9 \alpha_{0}^{11 / 3}(r)}, \\
& C_{3}(r)=\frac{-65 \frac{\alpha_{1}^{3}(r)}{\alpha_{0}^{5}(r)}+90 \frac{\alpha_{1}(r) \alpha_{2}(r)}{\alpha_{0}^{4}(r)}-27 \frac{\alpha_{3}(r)}{\alpha_{0}^{3}(r)}}{81 \alpha_{0}^{1 / 3}(r)} .
\end{aligned}
$$

These coefficients thus determine the expansion (77) for $m_{l}(r)$ in terms of $u_{l}(r)$.

\section{An Expression for the Position of the Turning Point in Terms of the Param- eters $u_{l}(a)$ and $\Lambda_{j}$}

Such an expansion can now be derived by applying (77) to $r=a$. We find

$$
m_{l}(a)=\gamma_{0}\left\{\frac{u_{l}(a)}{k_{0} a}\right\}^{1 / 3}+\gamma_{1}\left\{\frac{u_{l}(a)}{k_{0} a}\right\}^{3 / 3}+\gamma_{2}\left\{\frac{u_{l}(a)}{k_{0} a}\right\}^{5 / 3}+\gamma_{3}\left\{\frac{u_{l}(a)}{k_{0} a}\right\}^{7 / 3} \cdots
$$

where $\gamma_{j}=C_{j}(a)$ follows from a substitution of $r=a$ in (79). Introducing the new quantities $\beta_{j}=\alpha_{j}(a)$ we then have:

$$
\begin{aligned}
\gamma_{0} & =\frac{1}{\beta_{0}^{1 / 3}}, \\
\gamma_{1} & =\frac{-\beta_{1}}{3 \beta_{0}^{2}}, \\
\gamma_{2} & =\frac{4 \beta_{1}^{2}-3 \beta_{0} \beta_{2}}{9 \beta_{0}^{11 / 3}}, \\
\gamma_{3} & =\frac{-65 \frac{\beta_{1}^{3}}{\beta_{0}^{5}}+90 \frac{\beta_{1} \beta_{2}}{\beta_{0}^{4}}-27 \frac{\beta_{3}}{\beta_{0}^{3}}}{81 \beta_{0}^{1 / 3}} .
\end{aligned}
$$

Further, these coefficients $\beta_{j}$ are connected as follows with the profile, in view of $(76)$ for $r=a$,

$$
\beta_{j}=(-1)^{j} \frac{2}{3} \frac{2}{5} \frac{2}{7} \cdots \frac{2}{(2 j+3)}\left[\frac{d^{j}}{d\left\{M_{\mathrm{eff}}^{2}(r)\right\}^{j}} \frac{1}{r \frac{d M_{\mathrm{eff}}^{2}}{d r}}\right]_{r=a} .
$$

All derivatives occurring here can be expressed in terms of higher-order derivatives of $M^{2}$. For instance, we find:

$$
\begin{gathered}
\frac{d}{d M_{\mathrm{eff}}^{2}} \frac{1}{r \frac{d M_{\mathrm{eff}}^{2}}{d r}}=-\frac{1}{r^{2}\left(\frac{d M_{\mathrm{eff}}^{2}}{d r}\right)^{2}}-\frac{1}{r} \frac{\frac{d^{2} M_{\mathrm{eff}}^{2}}{d r^{2}}}{\left(\frac{d M_{\mathrm{eff}}^{2}}{d r}\right)^{3}}, \\
\frac{d^{2}}{\left(d M_{\mathrm{eff}}^{2}\right)^{2}} \frac{1}{r \frac{d M_{\mathrm{eff}}^{2}}{d r}}=\frac{2}{r^{3}\left(\frac{d M_{\mathrm{eff}}^{2}}{d r}\right)^{3}}+\frac{3}{r^{2}} \frac{\frac{d^{2} M_{\mathrm{eff}}^{2}}{d r^{2}}}{\left(\frac{d M_{\mathrm{eff}}^{2}}{d r}\right)^{4}}-\frac{1}{r} \frac{\frac{d^{3} M_{\mathrm{eff}}^{2}}{d r^{3}}}{\left(\frac{d M_{\mathrm{eff}}^{2}}{d r}\right)^{4}}+\frac{3}{r} \frac{\left\{\frac{d^{2} M_{\mathrm{eff}}^{2}}{d r^{2}}\right\}^{2}}{\left(\frac{d M_{\mathrm{eff}}^{2}}{d r}\right)^{5}} .
\end{gathered}
$$


We only need all these quantities at $r=a$ in order to evaluate the coefficients $\beta_{j}$ from (82). All terms then occurring can be expressed in terms of the profile constants $\Lambda_{j}$, by applying the definition (17). The first few prove to be given by:

$$
\begin{aligned}
& \beta_{0}=\frac{2}{3 \Lambda_{1}}, \\
& \beta_{1}=\frac{4}{15}\left(\frac{1}{\Lambda_{1}^{2}}+\frac{\Lambda_{2}}{\Lambda_{1}^{3}}\right), \\
& \beta_{2}=\frac{8}{105}\left(\frac{2}{\Lambda_{1}^{3}}+\frac{3 \Lambda_{2}-\Lambda_{3}}{\Lambda_{1}^{4}}+\frac{3 \Lambda_{2}^{2}}{\Lambda_{1}^{5}}\right), \\
& \beta_{3}=\frac{16}{945}\left(\frac{6}{\Lambda_{1}^{4}}+\frac{12 \Lambda_{2}-4 \Lambda_{3}+\Lambda_{4}}{\Lambda_{1}^{5}}+\frac{15 \Lambda_{2}^{2}-10 \Lambda_{2} \Lambda_{3}}{\Lambda_{1}^{6}}+\frac{15 \Lambda_{2}^{3}}{\Lambda_{1}^{7}}\right) .
\end{aligned}
$$

The coefficients $\gamma_{j}$ of the expansion (80) can thus be evaluated numerically by first computing the $\beta_{j}$ 's with the aid of (83) from the profile constants $\Lambda_{j}$, and next applying (81). For a further evaluation of the expansion (18) for the position $r_{l}$ of the turning point, we further have to determine the even powers of the expansion (80) in order to arrive at an explicit representation of $r_{l}$ in terms of both $\Lambda_{j}$ and $u_{l}(a)$. Obviously we then get an expansion of $\left(r_{l}-a\right) / a$ in terms of increasing integral powers of the quantity

$$
\left\{\frac{u_{l}(a)}{k_{0} a}\right\}^{2 / 3}
$$

with coefficients depending explicitly on both $\gamma_{j}$ and $\Lambda_{j}$. This rather tedious procedure leads to the following final representation:

$$
\frac{r_{l}-a}{a}=p_{1}\left\{\frac{u_{l}(a)}{k_{0} a}\right\}^{2 / 3}+p_{2}\left\{\frac{u_{l}(a)}{k_{0} a}\right\}^{4 / 3}+p_{3}\left\{\frac{u_{l}(a)}{k_{0} a}\right\}^{6 / 3}+p_{4}\left\{\frac{u_{l}(a)}{k_{0} a}\right\}^{8 / 3} \ldots
$$

the new coefficients of which can be derived as follows from the constants determined previously:

$$
\begin{aligned}
& p_{1}=-\frac{\gamma_{0}^{2}}{\Lambda_{1}}, \\
& p_{2}=-\frac{2 \gamma_{0} \gamma_{1}}{\Lambda_{1}}-\frac{\Lambda_{2}}{2 \Lambda_{1}^{3}} \gamma_{0}^{4}, \\
& p_{3}=-\frac{\gamma_{1}^{2}+2 \gamma_{0} \gamma_{2}}{\Lambda_{1}}-\frac{2 \Lambda_{2}}{\Lambda_{1}^{3}} \gamma_{0}^{3} \gamma_{1}+\left(\frac{\Lambda_{3}}{6 \Lambda_{1}^{4}}-\frac{\Lambda_{2}^{2}}{2 \Lambda_{1}^{5}}\right) \gamma_{0}^{6}, \\
& p_{4}=-2 \frac{\gamma_{0} \gamma_{3}+\gamma_{1} \gamma_{2}}{\Lambda_{1}}-\frac{\Lambda_{2}}{\Lambda_{1}^{3}}\left(3 \gamma_{0}^{2} \gamma_{1}^{2}+2 \gamma_{0}^{3} \gamma_{2}\right)+\left(\frac{\Lambda_{3}}{\Lambda_{1}^{4}}-\frac{3 \Lambda_{2}^{2}}{\Lambda_{1}^{5}}\right) \gamma_{0}^{5} \gamma_{1}+\left(\frac{5}{12} \frac{\Lambda_{2} \Lambda_{3}}{\Lambda_{1}^{6}}-\frac{5}{8} \frac{\Lambda_{2}^{3}}{\Lambda_{1}^{7}}-\frac{\Lambda_{4}}{24 \Lambda_{1}^{5}}\right) \gamma_{0}^{8} .
\end{aligned}
$$

Summarizing, all coefficients of the expansion (84) can be evaluated numerically by first deriving the $\beta_{j}$ 's from the profile parameters $\Lambda_{j}$ with the aid of (83), next the $\gamma_{j}$ 's from the $\beta_{j}$ 's with the aid of (81), and then the $p_{j}$ 's from the $\gamma_{j}$ 's with the aid of (85).

\section{Successive Developments Resulting in an Expansion of the Coefficients $B_{n}(I)$ in the Expansion (70) for the Dependence of $m_{l}(r)$ on $u_{l}(r)$}

Such an expansion is required for the subsequent analysis enabling the numerical evaluation of the eigenvalues and the corresponding expression of the field components. We start with a derivation of a series for the parameters $\eta_{l}^{(k)}$ of (73) in terms of the turningpoint coordinate $r_{l}$.

Let us first differentiate the square of (65). The definition of $\eta_{l}^{\prime}$ then involves 


$$
\eta_{l}^{\prime}=a \frac{d}{d r}\left[\frac{a^{2}}{r^{2}}\left\{M_{\text {eff }}^{2}(r)-C_{l}^{2}\right\}\right]_{r=r_{l}},
$$

which reduces, applying (14), to:

The Taylor expansion:

$$
\eta_{l}^{\prime}=\frac{a^{3}}{r_{l}^{2}}\left\{\frac{d M_{\text {eff }}^{2}(r)}{d r}\right\}_{r=r_{l}}
$$

$$
M_{\text {eff }}^{2}(r)=\sum_{j=0}^{\infty} \frac{\Lambda_{j}}{a^{j} j !}(r-a)^{j}
$$

may be used here for a further reduction which results in:

$$
\eta_{l}^{\prime}=\frac{a^{3}}{r_{l}^{2}} \sum_{j=0}^{\infty} \frac{\Lambda_{j}}{a^{j}(j-1) !}\left(r_{l}-a\right)^{j-1} .
$$

The higher-order coefficients $\eta_{l}^{(k)}$ are obtained in a similar way. For instance, by differentiating twice the square of (65) we obtain first:

$$
\eta_{l}^{\prime \prime}=a^{2} \frac{d^{2}}{d r^{2}}\left[\frac{a^{2}}{r^{2}}\left\{M_{\text {eff }}^{2}(r)-C_{l}^{2}\right\}\right]_{r=r_{l}}
$$

which can be reduced with the aid of (14) to:

$$
\eta_{l}^{\prime \prime}=a^{4}\left[-\frac{4}{r_{l}^{3}}\left\{\frac{d M_{\text {eff }}^{2}(r)}{d r}\right\}_{r=r_{l}}+\frac{1}{r_{l}^{2}}\left\{\frac{d^{2} M_{\text {eff }}^{2}(r)}{d r^{2}}\right\}_{r=r_{l}}\right] .
$$

When substituting the first and second derivative of (86) we arrive at:

$$
\eta_{l}^{\prime \prime}=a^{4}\left[-\frac{4}{r_{l}^{3}} \sum_{j=1}^{\infty} \frac{\Lambda_{j}}{a^{j}} \frac{\left(r_{l}-a\right)^{j-1}}{(j-1) !}+\frac{1}{r_{l}^{2}} \sum_{j=2}^{\infty} \frac{\Lambda_{j}}{a^{j}} \frac{\left(r_{l}-a\right)^{j-2}}{(j-2) !}\right] .
$$

By a continuation of this procedure we get expansions for all coefficients $\eta_{l}^{(k)}$ in terms of powers of $r_{l}-a$, divided by another power of $r_{l}$. In its turn, the latter power can be developed with the aid of the binominal expansion for

$$
\frac{1}{r_{l}^{k}}=\frac{1}{a^{k}}\left\{1+\frac{r_{l}-a}{a}\right\}^{-k}
$$

When assembling identical powers of $r_{l}-a$ we thus obtain expansions of the form:

$$
\eta_{l}^{(k)}=\sum_{j=0}^{\infty} q_{k j}\left\{\frac{r_{l}-a}{a}\right\}^{j},
$$

the lower-order coefficients of which prove to be given by:

$$
\begin{gathered}
q_{10}=\Lambda_{1} ; q_{11}=\Lambda_{2}-2 \Lambda_{1} ; q_{12}=3 \Lambda_{1}-2 \Lambda_{2}+\frac{\Lambda_{3}}{2} ; \\
q_{13}=-4 \Lambda_{1}+3 \Lambda_{2}-\Lambda_{3}+\frac{\Lambda_{4}}{6} ; \\
q_{14}=5 \Lambda_{1}-4 \Lambda_{2}+\frac{3}{2} \Lambda_{3}-\frac{\Lambda_{4}}{3}+\frac{\Lambda_{5}}{24} ; \\
q_{20}=-4 \Lambda_{1}+\Lambda_{2} ; q_{21}=12 \Lambda_{1}-6 \Lambda_{2}+\Lambda_{3} ; q_{22}=-24 \Lambda_{1}+15 \Lambda_{2}-4 \Lambda_{3}+\frac{\Lambda_{4}}{2} ; \\
q_{23}=40 \Lambda_{1}-28 \Lambda_{2}+9 \Lambda_{3}-\frac{5}{3} \Lambda_{4}+\frac{\Lambda_{5}}{6} ;
\end{gathered}
$$




$$
\begin{gathered}
q_{24}=-60 \Lambda_{1}+45 \Lambda_{2}-16 \Lambda_{3}+\frac{7}{2} \Lambda_{4}-\frac{\Lambda_{5}}{2}+\frac{\Lambda_{6}}{24} ; \\
q_{30}=18 \Lambda_{1}-6 \Lambda_{2}+\Lambda_{3} ; q_{31}=-72 \Lambda_{1}+36 \Lambda_{2}-8 \Lambda_{3}+\Lambda_{4} ; \\
q_{32}=180 \Lambda_{1}-108 \Lambda_{2}+30 \Lambda_{3}-5 \Lambda_{4}+\frac{\Lambda_{5}}{2} ; \\
q_{33}=-360 \Lambda_{1}+240 \Lambda_{2}-76 \Lambda_{3}+15 \Lambda_{4}-2 \Lambda_{5}+\frac{\Lambda_{6}}{6} ; \\
q_{34}=630 \Lambda_{1}-450 \Lambda_{2}+155 \Lambda_{3}-34 \Lambda_{4}+\frac{21}{4} \Lambda_{5}-\frac{7}{12} \Lambda_{6}+\frac{\Lambda_{7}}{24} ; \\
q_{40}=-96 \Lambda_{1}+36 \Lambda_{2}-8 \Lambda_{3}+\Lambda_{4} ; \\
q_{41}=480 \Lambda_{1}-240 \Lambda_{2}+60 \Lambda_{3}-10 \Lambda_{4}+\Lambda_{5} ; \\
q_{42}=-1440 \Lambda_{1}+840 \Lambda_{2}-240 \Lambda_{3}+45 \Lambda_{4}-6 \Lambda_{5}+\frac{\Lambda_{6}}{2} ; \\
q_{43}=3360 \Lambda_{1}-2160 \Lambda_{2}+680 \Lambda_{3}-140 \Lambda_{4}+21 \Lambda_{5}-\frac{7}{3} \Lambda_{6}+\frac{\Lambda_{7}}{6} ; \\
q_{44}=-6720 \Lambda_{1}+4620 \Lambda_{2}-1560 \Lambda_{3}+345 \Lambda_{4}-56 \Lambda_{5}+7 \Lambda_{6}-\frac{2}{3} \Lambda_{7}+\frac{\Lambda_{8}}{24}
\end{gathered}
$$

As a next step we may substitute in (87) the expansion (84) and the successive powers of this expansion. A new power series for $\eta_{l}^{(k)}$, viz

$$
\eta_{l}^{(k)}=\sum_{j=0}^{\infty} t_{k j}\left\{\frac{u_{l}(a)}{k_{0} a}\right\}^{\frac{2}{3} j}
$$

then results. Its lower-order coefficients can be represented as follows:

$$
\begin{aligned}
& t_{n 0}=q_{n 0} ; t_{n 1}=q_{n 1} p_{1} ; t_{n 2}=q_{n 1} p_{2}+q_{n 2} p_{1}^{2} ; \\
& t_{n 3}=q_{n 1} p_{3}+2 q_{n 2} p_{1} p_{2}+q_{n 3} p_{1}^{3} ; \\
& t_{n 4}=q_{n 1} p_{4}+q_{n 2}\left(p_{2}^{2}+2 p_{1} p_{3}\right)+3 q_{n 3} p_{1}^{2} p_{2}+g_{n 4} p_{1}^{4} .
\end{aligned}
$$

If these coefficients are also known we are enabled to derive also expansions of the coefficients $B_{k}^{(l)}$, defined by (74), in terms of the quantity $u_{l}(a) / k_{0} a$. For instance, we have

$$
\begin{aligned}
B_{0}^{(l)} & =\left(\frac{3}{2} \eta_{l}^{\prime}\right)^{1 / 3}=\left(\frac{3}{2}\right)^{1 / 3}\left[t_{10}+t_{11}\left\{\frac{u_{l}(a)}{k_{0} a}\right\}^{2 / 3}+t_{12}\left\{\frac{u_{l}(a)}{k_{0} a}\right\}^{4 / 3} \cdots\right]^{1 / 3} \\
& =\left(\frac{3}{2} t_{10}\right)^{1 / 3}\left[1+\frac{1}{3} \frac{t_{11}}{t_{10}}\left\{\frac{u_{l}(a)}{k_{0} a}\right\}^{2 / 3}+\left(\frac{1}{3} \frac{t_{12}}{t_{10}}-\frac{1}{9} \frac{t_{11}^{2}}{t_{10}^{2}}\right)\left\{\frac{u_{l}(a)}{k_{0} a}\right\}^{4 / 3} \cdots\right]
\end{aligned}
$$

and

$$
\begin{aligned}
B_{1}^{(l)} & =\frac{3}{10} \frac{\eta_{l}^{\prime \prime}}{\eta_{l}^{\prime}}=\frac{3}{10} \frac{t_{20}+t_{21}\left\{\frac{u_{l}(a)}{k_{0} a}\right\}^{2 / 3}+t_{22}\left\{\frac{u_{l}(a)}{k_{0} a}\right\}^{4 / 3} \cdots}{t_{10}+t_{11}\left\{\frac{u_{l}(a)}{k_{0} a}\right\}^{2 / 3}+t_{12}\left\{\frac{u_{l}(a)}{k_{0} a}\right\}^{4 / 3} \cdots} \\
& =\frac{3}{10} \frac{t_{20}}{t_{10}}\left[1+\left\{\frac{t_{21}}{t_{20}}-\frac{t_{11}}{t_{10}}\right\}\left\{\frac{u_{l}(a)}{k_{0} a}\right\}^{2 / 3}\right.
\end{aligned}
$$




$$
\left.+\left\{\frac{t_{11}^{2}}{t_{10}^{2}}-\frac{t_{21} t_{11}}{t_{20} t_{10}}+\frac{t_{22}}{t_{20}}-\frac{t_{12}}{t_{10}}\right\}\left\{\frac{u_{l}(a)}{k_{0} a}\right\}^{4 / 3} \cdots\right]
$$

and so on. In this way we get in general expansions of the form:

$$
B_{n}^{(l)}=\sum_{j=0}^{\infty} B_{n j}\left\{\frac{u_{l}(a)}{k_{0} a}\right\}^{2 / 3 j}
$$

The first few of these new coefficients are connected as follows with the former quantities $t_{n j}$ :

$$
\begin{aligned}
& B_{00}=\left(\frac{3}{2} t_{10}\right)^{\frac{1}{3}} ; B_{01}=\frac{t_{11}}{3 t_{10}}\left(\frac{3}{2} t_{10}\right)^{\frac{1}{3}} ; B_{02}=\left\{\frac{t_{12}}{3 t_{10}}-\frac{t_{11}^{2}}{9 t_{10}^{2}}\right\}\left(\frac{3}{2} t_{10}\right)^{\frac{1}{3}} ; \\
& B_{03}=\left\{\frac{t_{13}}{3 t_{10}}-\frac{2}{9} \frac{t_{11} t_{12}}{t_{10}^{2}}+\frac{5}{81} \frac{t_{11}^{3}}{t_{10}^{3}}\right\}\left(\frac{3}{2} t_{10}\right)^{\frac{1}{3}} \\
& B_{04}=\left\{\frac{t_{14}}{3 t_{10}}-\frac{t_{12}^{2}}{9 t_{10}^{2}}-\frac{2}{9} \frac{t_{11} t_{13}}{t_{10}^{2}}+\frac{5}{27} \frac{t_{12} t_{11}^{2}}{t_{10}^{3}}-\frac{10}{243} \frac{t_{11}^{4}}{t_{10}^{4}}\right\}\left(\frac{3}{2} t_{10}\right)^{\frac{1}{3}} \\
& B_{10}=\frac{3}{10} \frac{t_{20}}{t_{10}} ; B_{11}=\frac{3}{10}\left\{\frac{t_{21}}{t_{10}}-\frac{t_{11} t_{20}}{t_{10}^{2}}\right\} \text {; } \\
& B_{12}=\frac{3}{10}\left\{\frac{t_{22}}{t_{10}}-\frac{t_{11} t_{21}}{t_{10}^{2}}-\frac{t_{20} t_{12}}{t_{10}^{2}}+\frac{t_{20} t_{11}^{2}}{t_{10}^{3}}\right\} \\
& B_{13}=\frac{3}{10}\left\{\frac{t_{23}}{t_{10}}-\frac{t_{11} t_{22}}{t_{10}^{2}}-\frac{t_{12} t_{21}}{t_{10}^{2}}-\frac{t_{13} t_{20}}{t_{10}^{2}}+2 \frac{t_{11} t_{12} t_{20}}{t_{10}^{3}}+\frac{t_{21} t_{11}^{2}}{t_{10}^{3}}-\frac{t_{20} t_{11}^{3}}{t_{10}^{4}}\right\} ; \\
& B_{20}=\frac{9}{56}\left(\frac{2}{3 t_{10}}\right)^{\frac{1}{3}}\left\{\frac{t_{30}}{t_{10}}-\frac{19}{25} \frac{t_{20}^{2}}{t_{10}^{2}}\right\} \text {; } \\
& B_{21}=\frac{9}{56}\left(\frac{2}{3 t_{10}}\right)^{\frac{1}{3}}\left\{\frac{t_{31}}{t_{10}}-\frac{4}{3} \frac{t_{30} t_{11}}{t_{10}^{2}}-\frac{38}{25} \frac{t_{20} t_{21}}{t_{10}^{2}}+\frac{133}{75} \frac{t_{20}^{2} t_{11}}{t_{10}^{3}}\right\} ; \\
& B_{22}=\frac{9}{56}\left(\frac{2}{3 t_{10}}\right)^{\frac{1}{3}}\left\{\frac{4}{3} \frac{t_{30} t_{11}^{2}}{t_{10}^{3}}-\frac{t_{12} t_{30}}{t_{10}^{2}}-\frac{4}{3} \frac{t_{31} t_{11}}{t_{10}^{2}}+\frac{t_{32}}{t_{10}}\right. \\
& +\frac{38}{75} \frac{t_{11} t_{20} t_{21}}{t_{10}^{3}}-\frac{38}{75} \frac{t_{20}^{2} t_{11}^{2}}{t_{10}^{4}}-\frac{19}{25} \frac{\left(t_{21}-t_{20} \frac{t_{11}}{t_{10}}\right)^{2}}{t_{10}^{2}} \\
& +\frac{38}{25} \frac{t_{20}}{t_{10}^{2}}\left(\frac{t_{12} t_{20}}{t_{10}}-\frac{t_{11}^{2} t_{20}}{t_{10}^{2}}+\frac{t_{11} t_{21}}{t_{10}}-t_{22}\right) \\
& \left.+\left(\frac{2}{9} \frac{t_{11}^{2}}{t_{10}^{2}}-\frac{t_{12}}{3 t_{10}}\right)\left(\frac{t_{30}}{t_{10}}-\frac{19}{25} \frac{t_{20}^{2}}{t_{10}^{2}}\right)\right\} \\
& B_{30}=\left(\frac{2}{3 t_{10}}\right)^{2 / 3}\left\{\frac{t_{40}}{16 t_{10}}-\frac{t_{20} t_{30}}{7 t_{10}^{2}}+\frac{213}{2800} \frac{t_{20}^{3}}{t_{10}^{3}}\right\} ; \\
& B_{31}=\left(\frac{2}{3 t_{10}}\right)^{2 / 3}\left\{\frac{t_{41}}{16 t_{10}}-\frac{5}{48} \frac{t_{40} t_{11}}{t_{10}^{2}}-\frac{t_{20} t_{31}}{7 t_{10}^{2}}-\frac{t_{21} t_{30}}{7 t_{10}^{2}}+\frac{8}{21} \frac{t_{11} t_{20} t_{30}}{t_{10}^{3}}\right. \\
& \left.+\frac{639}{2800} \frac{t_{20}^{2} t_{21}}{t_{10}^{3}}-\frac{781}{2800} \frac{t_{11} t_{20}^{3}}{t_{10}^{4}}\right\}
\end{aligned}
$$

These coefficients, occurring in the expansion (90), determine the dependence of $m_{l}$ on $u_{l}$ according to (70). 


\section{Explicit Form of the Differential Equation (68)}

The expansion (70) for $m_{l}$ as a function of $u_{l}$ can also be differentiated in order to obtain corresponding expansions for $d m_{l} / d u_{l}$ and $d^{2} m_{l} / d u_{l}^{2}$. It is also possible to deduce from the latter developments of the quantities

$$
\frac{\left(\frac{d m_{l}}{d u_{l}}\right)^{2}}{m_{l}^{2}} \text { and } \frac{\frac{d^{2} m_{l}}{d u_{l}^{2}}}{m_{l}}
$$

occurring in (69). This elementary formal procedure leads to the following expansion for the coefficient $P_{l}$ of the final differential equation (68):

$$
P_{l}(u)=1+\frac{5}{36 u^{2}}+\sum_{n=0}^{\infty} \frac{C_{n}^{(l)}}{\left(k_{0} a\right)^{\frac{2}{3}(n+2)}} u^{\frac{2}{3}(n-1)} .
$$

Its first few coefficients read:

$$
\begin{aligned}
& C_{0}^{(l)}=\frac{B_{1}^{(l)^{2}}}{9 B_{0}^{(l)^{2}}}-\frac{4}{9} \frac{B_{2}^{(l)}}{B_{0}^{(l)}}, \\
& C_{1}^{(l)}=\frac{8}{9} \frac{B_{1}^{(l)} B_{2}^{(l)}}{B_{0}^{(l)^{2}}}-\frac{4}{3} \frac{B_{3}^{(l)}}{B_{0}^{(l)}}-\frac{2}{9} \frac{B_{1}^{(l)^{3}}}{B_{0}^{(l)^{3}}} \\
& C_{2}^{(l)}=-\frac{8}{3} \frac{B_{4}^{(l)}}{B_{0}^{(l)}}+\frac{8}{9} \frac{B_{2}^{(l)^{2}}}{B_{0}^{(l)^{2}}}+\frac{2 B_{1}^{(l)} B_{3}^{(l)}}{B_{0}^{(l)^{2}}}-\frac{14}{9} \frac{B_{1}^{(l)^{2}} B_{2}^{(l)}}{B_{0}^{(l)^{3}}}+\frac{1}{3} \frac{B_{1}^{(l)^{4}}}{B_{0}^{(l)}}, \\
& C_{3}^{(l)}=-\frac{40}{9} \frac{B_{5}^{(l)}}{B_{0}^{(l)}}+\frac{28}{9} \frac{B_{2}^{(l)} B_{3}^{(l)}}{B_{0}^{(l)^{2}}}+\frac{32}{9} \frac{B_{1}^{(l)} B_{4}^{(l)}}{B_{0}^{(l)^{2}}}-\frac{8}{3} \frac{B_{1}^{(l)} B_{2}^{(l)^{2}}}{B_{0}^{(l)^{3}}}-\frac{26}{9} \frac{B_{1}^{(l)^{2}} B_{3}^{(l)}}{B_{0}^{(l)^{3}}}+\frac{22}{9} \frac{B_{1}^{(l)^{3}} B_{2}^{(l)}}{B_{0}^{(l)^{4}}}-\frac{4}{9} \frac{\left.B_{1}^{(l)}\right)^{5}}{B_{0}^{(l)^{5}}},
\end{aligned}
$$

when derived straightforwardly with the aid of (70). In its turn the quantities $B_{k}^{(l)}$ are connected with the eigenvalue parameter $u_{l}(a)$ according to $(90)$. The later analysis requires the explicit dependence of the coefficients $C_{k}^{(l)}$ on this latter parameter. This dependence is obtained by determining the powers of the expansion (90) and also the relevant fractions resulting from the divisions of such powers.

The evaluation of this procedure leads to further expansions of the form:

$$
C_{k}^{(l)}=\sum_{j=0}^{\infty} C_{k j}\left\{\frac{u_{l}(a)}{k_{0} a}\right\}^{\frac{2}{3} j}
$$

the new coefficients of which may be computed by performing the mentioned procedure numerically, without applying the complicated analytical expressions which connect the $C_{k j}$ 's with the coefficients $B_{n j}$ determined previously. However, for convenience we give the expressions for the simplest coefficients $C_{k j}$ :

$$
\begin{aligned}
C_{00} & =-\frac{4}{9} \frac{B_{20}}{B_{00}}+\frac{1}{9} \frac{B_{10}^{2}}{B_{00}^{2}} \\
C_{01} & =-\frac{4}{9} \frac{B_{21}}{B_{00}}+\frac{4 B_{20} B_{01}+2 B_{10} B_{11}}{9 B_{00}^{2}}-\frac{2}{9} \frac{B_{01} B_{10}^{2}}{B_{00}^{3}}, \\
C_{02} & =-\frac{4}{9} \frac{B_{22}}{B_{00}}+\frac{4\left(B_{21} B_{01}+B_{20} B_{02}\right)+2 B_{10} B_{12}+B_{11}^{2}}{9 B_{00}^{2}}-\frac{4 B_{20} B_{01}^{2}+B_{10}\left(4 B_{01} B_{11}+2 B_{10} B_{02}\right)}{9 B_{00}^{3}}+\frac{B_{10}^{2} B_{01}^{2}}{3 B_{00}^{4}}, \\
10 & =-\frac{4}{3} \frac{B_{30}}{B_{00}}+\frac{8}{9} \frac{B_{10} B_{20}}{B_{00}^{2}}-\frac{2}{9} \frac{B_{10}^{3}}{B_{00}^{3}},
\end{aligned}
$$$$
609993-62-4
$$ 


$$
\begin{aligned}
& C_{11}=-\frac{4}{3} \frac{B_{31}}{B_{00}}+\frac{\frac{4}{3} B_{30} B_{01}+\frac{8}{9} B_{10} B_{21}+\frac{8}{9} B_{11} B_{20}}{B_{00}^{2}}-\frac{\frac{2}{3} B_{10}^{2} B_{11}+\frac{16}{9} B_{01} B_{10} B_{20}}{B_{00}^{3}}+\frac{2}{3} \frac{B_{10}^{3} B_{01}}{B_{00}^{4}}, \\
& C_{20}=-\frac{8}{3} \frac{B_{40}}{B_{00}}+\frac{2 B_{10} B_{30}+\frac{8}{9} B_{20}^{2}}{B_{00}^{2}}-\frac{14}{9} \frac{B_{10}^{2} B_{20}}{B_{00}^{3}}+\frac{1}{3} \frac{B_{10}^{4}}{B_{00}^{4}} .
\end{aligned}
$$

\section{Solution of the Differential Equation (68)}

This equation depends completely on the function $P_{l}$ which is represented by (92) as a power series in $\left(k_{0} a\right)^{-2 / 3}$. The only term remaining for $k_{0} a \rightarrow \infty$ involves the solution $G(u)$ $=\sqrt{u} H_{1 / 3}^{(1)}(u)$ known from the "extended W.K.B. approximation." The next terms may be considered as corrections for large finite $k_{0} a$ and suggest the following representation for the complete solution:

$$
G(u)=\sum_{n=0}^{\infty} \frac{g_{n}(u)}{\left(k_{0} a\right)^{2 n / 3}}
$$

We substitute this series into (68) and assemble the contributions proportional to one and the same power of $\left(k_{0} a\right)^{-2 / 3}$. It leads to the following set of equations:

$$
\begin{aligned}
& \frac{d^{2} g_{0}}{d u^{2}}+\left(1+\frac{5}{36 u^{2}}\right) g_{0}=0 \\
& \frac{d^{2} g_{1}}{d u^{2}}+\left(1+\frac{5}{36 u^{2}}\right) g_{1}=0 \\
& \frac{d^{2} g_{n}}{d u^{2}}+\left(1+\frac{5}{36 u^{2}}\right) g_{n}=\sum_{m=0}^{n-2} C_{n-m-2} u^{(2 / 3)(n-m-3)} g_{m}(u) . \quad(n \geq 2)
\end{aligned}
$$

Starting from the normalized "extended W.K.B. approximation" $g_{0}(u)=\sqrt{u} H_{1 / 3}^{(1)}(u)$, satisfying the first equation, we may take $g_{1}=0$. Taking into account these special values of $g_{0}$ and $g_{1}$, we find the next equations:

$$
\begin{aligned}
& \left(\frac{d^{2}}{d u^{2}}+1+\frac{5}{36 u^{2}}\right) g_{2}=C_{0} u^{-1 / 6} H_{1 / 3}^{(1)}(u), \\
& \left(\frac{d^{2}}{d u^{2}}+1+\frac{5}{36 u^{2}}\right) g_{3}=C_{1} \sqrt{u} H_{1 / 3}^{(1)}(u), \\
& \left(\frac{d^{2}}{d u^{2}}+1+\frac{5}{36 u^{2}}\right) g_{4}=C_{2} u^{7 / 6} H_{1 / 3}^{(1)}(u)+C_{0} u^{-2 / 3} g_{2}(u),
\end{aligned}
$$

etc. All these equations can be solved easily in succession, by substituting for the unknown function in any further equation the sum of (a) the Hankel function $H_{1 / 3}^{(1)}(u)$ multiplied by a finite number of (positive or negative) powers of $u^{1 / 6}$ with indefinite constant factors, (b) the Hankel function $H_{2 / 3}^{(1)}(u)$ multiplied by another number of such powers. The powers to be introduced here, as well as the coefficients in front of them, can be determined conveniently by applying the following identity:

$$
\begin{aligned}
\left(\frac{d^{2}}{d u^{2}}+1+\right. & \left.\frac{5}{36 u^{2}}\right)\left\{\alpha_{n} u^{\frac{2}{3} n+\frac{1}{2}} H_{1 / 3}^{(1)}(u)+\beta_{m} u^{\frac{2}{3} m+{ }^{5}} H_{2 / 3}^{(1)}(u)\right\}=\left\{\frac{4}{9}\left(n^{2}-n\right) \alpha_{n} u^{\frac{2}{3} n-\frac{3}{2}}\right. \\
& \left.+\frac{2}{3} e^{i \pi / 3}(2 m+1) \beta_{m} u^{\frac{2}{3} m-\frac{1}{6}}\right\} H_{1 / 3}^{(1)}(u)+\left\{-\frac{4}{3} e^{-i \pi / 3} n \alpha_{n} u^{\frac{2}{3} n-\frac{1}{2}}+\frac{4}{9}\left(m^{2}-m\right) \beta_{m} u^{\frac{2}{3} m-\frac{7}{6}}\right\} H_{2 / 3}^{(1)}(u) .
\end{aligned}
$$


The resulting functions $g_{n}$, substituted into (96), yield the following first terms for the complete solution of (68) that starts with the "extended W.K.B. approximation":

$$
\begin{aligned}
& G(u)= \sqrt{u} H_{1 / 3}^{(1)}(u)+\frac{3}{2} e^{-i \pi / 3} \frac{C_{0} u^{5 / 6} H_{2 / 3}^{(1)}(u)}{\left(k_{0} a\right)^{4 / 3}}+\frac{C_{1}}{2} \frac{\frac{2}{3} \sqrt{u} H_{1 / 3}^{(1)}(u)+e^{-i \pi / 3} u^{3 / 2} H_{2 / 3}^{(1)}(u)}{\left(k_{0} a\right)^{2}} \\
&+\frac{\left(-\frac{9}{8} C_{0}^{2}+\frac{C_{2}}{5}\right) u^{7 / 6} H_{1 / 3}^{(1)}(u)+\frac{3}{10} e^{-i \pi / 3} C_{2} u^{13 / 6} H_{2 / 3}^{(1)}(u)}{\left(k_{0} a\right)^{8 / 3}} \\
&+\frac{\left(\frac{3}{14} C_{3}-\frac{3}{4} C_{0} C_{1}\right) u^{11 / 6} H_{1 / 3}^{(1)}(u)+e^{-i \pi / 3}\left\{\left(\frac{3}{2} C_{0} C_{1}-\frac{2}{7} C_{3}\right) u^{5 / 6}+\frac{3}{14} C_{3} u^{17 / 6}\right\} H_{2 / 3}^{(1)}(u)}{\left(k_{0} a\right)^{10 / 3}} \ldots
\end{aligned}
$$

\section{Equation for the Eigenvalues in Terms of the Functions $G$ and $m_{l}$}

This equation results from the boundary condition (23) holding at the earth's surface $(r=a)$. It reads as follows for the individual modes $\Pi_{l}=f_{l}(r) P_{l}(\cos \theta)$ :

$$
\frac{\partial}{\partial r}\left(r f_{l}\right)=\frac{\Gamma}{a} r f_{l} \text { at } r=a .
$$

In view of the definition (67) for the function $G$ this proves to be equivalent to:

$$
\frac{1}{G} \frac{\partial G}{\partial r}-\frac{1}{2} \frac{\frac{d m_{l}}{d r}}{m_{l}}=\frac{\Gamma}{a} \text { at } r=a .
$$

We shall pass from $r$ to the variable $u$ which can be performed with the relation

$$
\frac{d u_{l}}{d r}=k_{0} m_{l}
$$

resulting from (66). The equation (98) then transforms into:

$$
\frac{1}{G} \frac{d G}{d u} k_{0} a m_{l}-\frac{k_{0} a}{2} \frac{d m_{l}}{d u}=\Gamma \text { at } r=a .
$$

\section{An Explicit Form for the Equation for the Eigenvalues}

The function $G(u)$ is represented explicitly by (97). Its derivative $d G / d u$ can also be expressed in terms of the functions $H_{1 / 3}$ and $H_{2 / 3}$, using the identities:

$$
\begin{aligned}
& H_{1 / 3}^{(1) \prime}(u)=-\frac{1}{3 u} H_{1 / 3}^{(1)}(u)-e^{-i \pi / 3} H_{2 / 3}^{(1)}(u), \\
& H_{2 / 3}^{(1) \prime}(u)=-\frac{2}{3 u} H_{2 / 3}^{(1)}(u)+e^{i \pi / 3} H_{1 / 3}^{(1)}(u) .
\end{aligned}
$$

We thus find the following expansion corresponding to (97):

$$
\begin{aligned}
\frac{d G}{d u}=\left\{\frac{1}{6 \sqrt{u}} H_{1 / 3}^{(1)}(u)-e^{-i \pi / 3} \sqrt{u} H_{2 / 3}^{(1)}(u)\right\} \\
+\frac{3}{2} C_{0} \frac{u^{\frac{5}{6}} H_{1 / 3}^{(1)}(u)+e^{-i \pi / 3} \frac{u^{-1 / 6}}{6} H_{2 / 3}^{(1)}(u)}{\left(k_{0} a\right)^{4 / 3}}+\frac{C_{1}}{2} \frac{\left(\frac{1}{9 \sqrt{u}}+u^{3 / 2}\right) H_{1 / 3}^{(1)}(u)+e^{-i \pi / 3} \frac{\sqrt{u}}{6} H_{2 / 3}^{(1)}(u)}{\left(k_{0} a\right)^{2}} \\
+\frac{\left\{\left(-\frac{15}{16} C_{0}^{2}+\frac{C_{2}}{6}\right) u^{1 / 6}+\frac{3}{10} C_{2} u^{13 / 6}\right\} H_{1 / 3}^{(1)}(u)+e^{-i \pi / 3}\left(\frac{9}{8} C_{0}^{2}+\frac{C_{2}}{4}\right) u^{7 / 6} H_{2 / 3}^{(1)}(u)}{\left(k_{0} a\right)^{8 / 3}}+\ldots
\end{aligned}
$$


A division of (97) by this new expansion leads to the following corresponding representation for $(d G / d u) / G$ :

$$
\begin{aligned}
\frac{d G}{G}=\left(\frac{1}{6 u}-e^{-i \pi / 3} \frac{H_{2 / 3}^{(1)}(u)}{H_{1 / 3}^{(1)}(u)}\right)+\frac{3}{2} C_{00} \frac{u^{1 / 3}\left\{1-e^{i \pi / 3} \frac{H_{2 / 3}^{(1)}(u)}{H_{1 / 3}^{(1)}(u)}\right\}}{\left(k_{0} a\right)^{4 / 3}} & \\
+ & \frac{\frac{u}{2}\left(3 C_{01}+C_{10}\right)+\frac{e^{i \pi / 3}}{3} C_{10} \frac{H_{2 / 3}^{(1)}(u)}{H_{1 / 3}^{(1)}(u)}-e^{i \pi / 3}\left(\frac{3}{2} C_{01}^{\gamma}+\frac{C_{10}}{2}\right) u \frac{H_{2 / 3}^{(1)}(u)^{2}}{H_{1 / 3}^{(1)}(u)^{2}}}{\left(k_{0} a\right)^{2}} \\
+ & +\frac{\left(-\frac{3}{4} C_{00}^{2}+\frac{2}{15} C_{20}\right) u^{-\frac{1}{3}}+\left(\frac{3}{10} C_{20}+\frac{3}{2} C_{02}+\frac{C_{11}}{2}\right) u^{5 / 3}\left\{1-e^{i \pi / 3} \frac{H_{2 / 3}^{(1)}(u)}{H_{1 / 3}^{(1)}(u)}\right\}}{\left(k_{0} a\right)^{8 / 3}} \\
& +\frac{\left.9 e^{-i \pi / 3}\left(\frac{2}{5} C_{20}-\frac{9}{4} C_{00}^{2}\right)+\frac{e^{i \pi / 3} C_{11}}{3}\right\} u^{2 / 3} \frac{H_{2 / 3}^{(1)}(u)}{H_{1 / 3}^{(1)}(u)}+\frac{9}{4} C_{00}^{2} u^{2 / 3} \frac{H_{2 / 3}^{(1)}(u)}{H_{1 / 3}^{(1)}(u)}}{\left(k_{0} a\right)^{8 / 3}}
\end{aligned}
$$

In order to evaluate the first term of (99) the latter expansion has to be multiplied by that of $k_{0} a m_{l}$ which, in its turn, is obtained (in the special case $r=a$ ) with the aid of (70) and (90). We find, for each eigenvalue $l$

$$
\begin{aligned}
k_{0} a m(u) & =\left(k_{0} a\right)^{2 / 3} B_{00} u^{1 / 3}+\left(B_{01}+B_{10}\right) u+\left(B_{02}+B_{11}+B_{20}\right) \frac{u^{5 / 3}}{\left(k_{0} a\right)^{2 / 3}} \\
& +\left(B_{03}+B_{12}+B_{21}+B_{30}\right) \frac{u^{7 / 3}}{\left(k_{0} a\right)^{4 / 3}}+\left(B_{04}+B_{13}+B_{22}+B_{31}+B_{40}\right) \frac{u^{3}}{\left(k_{0} a\right)^{2}} \ldots \text { for } u=u_{l}(\boldsymbol{a}) .
\end{aligned}
$$

The product constituting the first term of (99) can thus also be expanded with respect to $\left(k_{0} a\right)^{-2 / 3}$. The corresponding expansion of $d m_{l} / d u$, entering in the next term, follows from the derivative of (70); it depends on the same coefficients $B_{n j}$ of (90) since we have to take $r=a$ throughout. By these procedures we arrive at the following final form for the equation (99):

$$
\begin{aligned}
& \Gamma=-e^{-i \pi / 3} B_{00} u^{1 / 3} \frac{H_{223}^{(1)}(u)}{H_{1 / 3}^{(1)}(u)}\left(k_{0} a\right)^{2 / 3}+\left\{a_{00}+a_{01} e^{-i \pi / 3} u \frac{H_{2 / 3}^{(1)}(u)}{H_{1 / 3}^{(1)}(u)}\right\} \\
&+ \frac{u^{2 / 3}\left\{a_{10}+e^{-i \pi / 3} a_{11} u \frac{H_{2 / 3}^{(1)}(u)}{H_{1 / 3}^{(1)}(u)}+e^{i \pi / 3} a_{12} \frac{H_{2 / 3}^{(1)}(u)}{H_{1 / 3}^{(1)}(u)}\right\}}{\left(k_{0} a\right)^{2 / 3}} \\
&+\frac{u^{4 / 3}\left\{a_{20}+\left(\frac{e^{i \pi / 3} a_{21}}{u}+e^{-i \pi / 3} a_{22} u\right) \frac{H_{2 / 3}^{(1)}(u)}{H_{1 / 3}^{(1)}(u)}+e^{i \pi / 3} a_{23} \frac{H_{2 / 3}^{(1)}(u)}{H_{1 / 3}^{(1)}(u)}\right\}}{\left(k_{0} a\right)^{4 / 3}} \\
&+\frac{a_{30}+a_{31} u^{2}+\left(e^{-i \pi / 3} a_{32}+e^{i \pi / 3} a_{33}\right) u \frac{H_{2 / 3}^{(1)}(u)}{H_{1 / 3}^{(1)}(u)}+e^{-i \pi / 3} a_{34} u^{3} \frac{H_{2 / 3}^{(1)}(u)}{H_{1 / 3}^{(1)}(u)}}{\left(k_{0} a\right)^{2}} \\
&+\frac{+e^{i \pi / 3} a_{35} u^{2} \frac{H_{213}^{(1)}{ }^{2}(u)}{H_{1 / 3}^{(1)}(u)}+a_{36} u \frac{H_{23 / 3}^{(1)}(u)}{H_{1 / 3}^{(1)}(u)}}{\left(k_{0} a\right)^{2}}+\cdots,
\end{aligned}
$$

in which $u$ is short for $u_{l}(a)$ whereas the new numerical coefficients introduced here are defined by: 


$$
\begin{aligned}
& a_{00}=\frac{-B_{10}}{3} ; a_{01}=-\left(B_{01}+B_{10}\right), \\
& a_{10}=\frac{3}{2} C_{00} B_{00}-\frac{B_{11}}{3}-\frac{2}{3} B_{20}, \\
& a_{11}=-\left(B_{02}+B_{11}+B_{20}\right), \\
& a_{12}=-\frac{3}{2} C_{00} B_{00} \\
& a_{20}=\frac{B_{00}}{2}\left(3 C_{01}+C_{10}\right)+\frac{3}{2}\left(B_{01}+B_{10}\right) C_{00}-\frac{B_{12}}{3}-\frac{2}{3} B_{21}-B_{: 0}, \\
& a_{21}=\frac{1}{3} B_{00} C_{10}, \\
& a_{22}=-\left(B_{03}+B_{12}+B_{21}+B_{30}\right), \\
& a_{23}=-\left\{B_{00}\left(\frac{3}{2} C_{01}+\frac{C_{10}}{2}\right)+\frac{3}{2}\left(B_{01}+B_{10}\right) C_{00}\right\}, \\
& a_{30}=B_{00}\left(-\frac{3}{4} C_{00}^{2}+\frac{2}{15} C_{20}\right) \text {, } \\
& a_{31}=B_{00}\left(\frac{3}{10} C_{20}+\frac{3}{2} C_{02}+\frac{C_{11}}{2}\right)+\frac{\left(B_{01}+B_{10}\right)}{2}\left(3 C_{01}+C_{10}\right)+\frac{3}{2}\left(B_{02}+B_{11}+B_{20}\right) C_{00} \\
& -\frac{1}{3}\left(B_{13}+2 B_{22}+3 B_{31}+4 B_{40}\right), \\
& a_{32}=B_{00}\left(\frac{2}{5} C_{20}-\frac{9}{4} C_{00}^{2}\right) \\
& a_{33}=\frac{B_{00} C_{11}+\left(B_{01}+B_{10}\right) C_{10}}{3}, \\
& a_{34}=-\left(B_{04}+B_{13}+B_{22}+B_{31}+B_{40}\right), \\
& a_{35}=-\left[B_{00}\left(\frac{3}{10} C_{20}+\frac{C_{11}}{2}+\frac{3}{2} C_{02}\right)+\left(B_{01}+B_{10}\right)\left(\frac{3}{2} C_{01}+\frac{C_{10}}{2}\right)+\frac{3}{2}\left(B_{02}+B_{11}+B_{20}\right) C_{00}\right], \\
& a_{36}=\frac{9}{4} B_{00} C_{00}^{2} .
\end{aligned}
$$

The equation (101) fixes the eigenvalues $l$ of our problem since it determines the value of

$$
u_{l}(a)=k_{0} a \int_{r_{l}}^{a} \frac{d s}{s} \sqrt{M_{\mathrm{eff}}^{2}(s)-C_{l}^{2}}
$$

which leads to a well defined value of $C_{l}=\sqrt{l(l+1)} / k_{0} a$. This will be discussed further in section 26.

\section{Solution of the Equation for the Eigenvalues}

A first approximative solution of this equation is obtained when neglecting all terms but the first in the right-hand side of (101). The corresponding value $u_{0}$ of $u_{l}(a)$ satisfies the equation 


$$
\Gamma^{\prime}=-e^{-i \pi / 3} B_{00} u_{0}^{1 / 3} \frac{H_{2 / 3}^{(1)}\left(u_{0}\right)}{H_{1 / 3}^{(1)}\left(u_{0}\right)}
$$

if we put $\Gamma=\left(k_{0} a\right)^{2 / 3} \Gamma^{\prime}$. In the case of a homogeneous atmosphere the relation (104) constitutes the base for the conventional diffraction theory for the propagation of radio waves round a smooth spherical earth.

The decrease proportional to powers of $\left(k_{0} a\right)^{-2 / 3}$ of the next terms in the right-hand side of (101) suggests to expand the rigorous solution as follows, for each eigenvalue $u=u_{l}(a)$.

$$
u=u_{0}+\frac{u_{1}}{\left(k_{0} a\right)^{2 / 3}}+\frac{u_{2}}{\left(k_{0} a\right)^{4 / 3}}+\frac{u_{3}}{\left(k_{0} a\right)^{2}} \ldots
$$

It is then necessary to expand all coefficients in (101) accordingly, with the aid of Taylor series around $u=u_{0}$. This will be indicated in detail for the first coefficient, viz,

$\frac{u^{1 / 3} H_{2 / 3}^{(1)}(u)}{H_{1 / 3}^{(1)}(u)}=\frac{u_{0}^{1 / 3} H_{2 / 3}^{(1)}\left(u_{0}\right)}{H_{1 / 3}^{(1)}\left(u_{0}\right)}+\frac{d}{d u}\left\{\frac{u^{1 / 3} H_{2 / 3}^{(1)}(u)}{H_{1 / 3}^{(1)}(u)}\right\}_{u=u_{0}}\left(u-u_{0}\right)+\frac{1}{2 !} \frac{d^{2}}{d u^{2}}\left\{\frac{u^{1 / 3} H_{2 / 3}^{(1)}(u)}{H_{1 / 3}^{(1)}(u)}\right\}_{u=u_{0}}\left(u-u_{0}\right)^{2} \ldots$

After substitution of (105) we get an expansion with respect to $\left(k_{0} a\right)^{-2 / 3}$ that starts with:

$$
\begin{aligned}
\frac{u^{1 / 3} H_{2 / 3}^{(1)}(u)}{H_{1 / 3}^{(1)}(u)}=\frac{u_{0}^{1 / 3} H_{2 / 3}^{(1)}\left(u_{0}\right)}{H_{1 / 3}^{(1)}\left(u_{0}\right)} & +\frac{1}{\left(k_{0} a\right)^{2 / 3}} u_{1} \frac{d}{d u}\left\{\frac{u^{1 / 3} H_{2 / 3}^{(1)}(u)}{H_{1 / 3}^{(1)}(u)}\right\}_{u=u_{0}} \\
+ & \frac{1}{\left(k_{0} a\right)^{4 / 3}}\left(\frac{u_{1}^{2}}{2} \frac{d^{2}}{d u^{2}}+u_{2} \frac{d}{d u}\right)\left\{\frac{u^{1 / 3} H_{2 / 3}^{(1)}(u)}{H_{1 / 3}^{(1)}(u)}\right\}_{u=u_{0}} \\
& +\frac{1}{\left(k_{0} a\right)^{2}}\left(\frac{u_{1}^{3}}{6} \frac{d^{3}}{d u^{3}}+u_{1} u_{2} \frac{d^{2}}{d u^{2}}+u_{3} \frac{d}{d u}\right)\left\{\frac{u^{1 / 3} H_{2 / 3}^{(1)}(u)}{H_{1 / 3}^{(1)}(u)}\right\}_{u=u_{0}}+\ldots
\end{aligned}
$$

All higher-order derivatives occurring here can be expressed in terms of the Hankel functions of the orders $1 / 3$ and $2 / 3$, by applying a number of times the elementary rules known from the theory of Bessel functions. For the evaluation of the present coefficients we need the relations

$$
\begin{aligned}
\frac{d}{d u} \frac{u^{1 / 3} H_{2 / 3}^{(1)}(u)}{H_{1 / 3}^{(1)}(u)}= & u^{1 / 3}\left\{e^{i \pi / 3}+e^{-i \pi / 3} \frac{H_{2 / 3}^{(1)}{ }^{2}(u)}{H_{1 / 3}^{(1)}(u)}\right\}, \\
\frac{d^{2}}{d u^{2}} \frac{u^{1 / 3} H_{2 / 3}^{(1)}(u)}{H_{1 / 3}^{(1)}(u)}= & \frac{e^{i \pi / 3}}{3 u^{2 / 3}}+2 u^{1 / 3} \frac{H_{2 / 3}^{(1)}(u)}{H_{1 / 3}^{(1)}(u)}-\frac{e^{-i \pi / 3}}{\partial u^{2 / 3}} \frac{H_{2 / 3}^{(1)}(u)}{H_{1 / 3}^{(1)}(u)}-2 e^{i \pi / 3} u^{1 / 3} \frac{H_{2 / 3}^{(1)}(u)^{3}}{H_{1 / 3}^{(1)}(u)^{3}}, \\
\frac{d^{3}}{d u^{3}} \frac{u^{1 / 3} H_{2 / 3}^{(1)}(u)}{H_{1 / 3}^{(1)}(u)}= & 2 e^{i \pi / 3}\left(u^{1 / 3}-\frac{1}{9 u^{5 / 3}}\right)-\frac{2}{3 u^{2 / 3}} \frac{H_{2 / 3}^{(1)}(u)}{H_{1 / 3}^{(1)}(u)}+4 e^{-i \pi / 3}\left(2 u^{1 / 3}+\frac{1}{9 u^{5 / 3}}\right) \frac{H_{2 / 3}^{(1)}{ }^{2}(u)}{H_{1 / 3}^{(1)}{ }^{2}(u)} \\
& +\frac{2 e^{i \pi / 3}}{u^{2 / 3}} \frac{H_{2 / 3}^{(1)}(u)}{H_{1 / 3}^{(1)}(u)}-6 u^{1 / 3} \frac{H_{2 / 3}^{(1)}(u)}{H_{1 / 3}^{(1)}(u)} .
\end{aligned}
$$

In all these coefficients we may substitute, in view of (104),

$$
\frac{H_{2 / 3}^{(1)}\left(u_{0}\right)}{H_{1 / 3}^{(1)}\left(u_{0}\right)}=-\frac{e^{i \pi / 3}}{B_{00}} \frac{\Gamma^{\prime}}{u_{0}^{1 / 3}}
$$

In this way we get the following expansion replacing (106):

$\frac{u^{1 / 3} H_{2 / 3}^{(1)}(u)}{H_{1 / 3}^{(1)}(u)}=-\frac{e^{i \pi / 3}}{B_{00}} \Gamma^{\prime}+e^{i \pi / 3} \frac{u_{1} u_{0}^{1 / 3}\left(1+\frac{\Gamma^{\prime 2}}{B_{00}^{2} u_{0}^{2 / 3}}\right)}{\left(k_{0} a\right)^{2 / 3}}$ 


$$
\begin{aligned}
& +e^{i \pi / 3} \frac{\left(u_{2} u_{0}^{1 / 3}+\frac{u_{1}^{2}}{6 u_{0}^{2 / 3}}\right)-\frac{\Gamma^{\prime}}{B_{00}} u_{1}^{2}+\frac{\Gamma^{\prime 2}}{B_{00}^{2}}\left(\frac{u_{2}}{u_{0}^{1 / 3}}-\frac{u_{1}^{2}}{6 u_{0}^{4 / 3}}\right)-\frac{\Gamma^{\prime 3} u_{1}^{2}}{B_{00}^{3} u_{0}^{2 / 3}}}{\left(k_{0} a\right)^{4 / 3}} \\
& +e^{i \pi / 3} \frac{\left(u_{3} u_{0}^{1 / 3}+\frac{u_{1} u_{2}}{3 u_{0}^{2 / 3}}+\frac{u_{1}^{3}}{3} u_{0}^{1 / 3}-\frac{u_{1}^{3}}{27 u_{0}^{5 / 3}}\right)-\frac{\Gamma^{\prime}}{B_{00}}\left(2 u_{1} u_{2}-\frac{u_{1}^{3}}{9 u_{0}}\right)+\frac{\Gamma^{\prime 2}}{B_{00}^{2}}\left(\frac{u_{3}}{u_{0}^{1 / 3}}-\frac{u_{1} u_{2}}{3 u_{0}^{4 / 3}}+\frac{4}{3} \frac{u_{1}^{3}}{u_{0}^{1 / 3}}+\frac{2}{27} \frac{u_{1}^{3}}{u_{0}^{7 / 3}}\right)}{\left(k_{0} a\right)^{2}} \\
& +e^{i \pi / 3} \frac{+\frac{\Gamma^{\prime 3}}{B_{00}^{3}}\left(-\frac{2 u_{1} u_{2}}{u_{0}^{2 / 3}}+\frac{u_{1}^{3}}{3 u_{0}^{5 / 3}}\right)+\frac{\Gamma^{\prime 4}}{B_{00}^{4}} \frac{u_{1}^{3}}{u_{0}}}{\left(k_{0} a\right)^{2}}+\ldots
\end{aligned}
$$

Corresponding expansions are found for the other quantities entering in (101). They start with:

$$
\begin{aligned}
& u \frac{H_{2 / 3}^{(1)}(u)}{H_{1 / 3}^{(1)}(u)}=-e^{i \pi / 3} \frac{\Gamma^{\prime} u_{0}^{2 / 3}}{B_{00}}+e^{i \pi / 3} \frac{u_{1}\left(u_{0}-\frac{2}{3} \frac{\Gamma^{\prime}}{B_{00} u_{0}^{1 / 3}}+\frac{\Gamma^{\prime 2} u_{0}^{1 / 3}}{B_{00}^{2}}\right)}{\left(k_{0} a\right)^{2 / 3}} \\
& +e^{i \pi / 3} \frac{\left(u_{2} u_{0}+\frac{5}{6} u_{1}^{2}\right)-\frac{\Gamma^{\prime}}{B_{00} u_{0}^{1 / 3}}\left\{\frac{2}{3} u_{2}+u_{1}^{2}\left(u_{0}-\frac{1}{9 u_{0}}\right)\right\}}{\left(k_{0} a\right)^{4 / 3}} \\
& +e^{i \pi / 3} \frac{\frac{\Gamma^{\prime 2}}{B_{00}^{2} u_{0}^{2 / 3}}\left(u_{2} u_{0}+\frac{u_{1}^{2}}{2}\right)-\frac{\Gamma^{\prime 3}}{B_{00}^{3}} u_{1}^{2}}{\left(k_{0} a\right)^{4 / 3}}+\ldots, \\
& u^{2 / 3}=u_{0}^{2 / 3}+\frac{2}{3} \frac{u_{1} u_{0}^{-1 / 3}}{\left(k_{0} a\right)^{2 / 3}}+\ldots \\
& u^{5 / 3} \frac{H_{2 / 3}^{(1)}(u)}{H_{1 / 3}^{(1)}(u)}=-e^{i \pi / 3} \frac{\Gamma^{\prime}}{B_{00}} u_{0}^{4 / 3}+e^{i \pi / 3} \frac{u_{1}\left(u_{0}^{5 / 3}-\frac{4}{3} \frac{\Gamma^{\prime}}{B_{00}} u_{0}^{1 / 3}+\frac{\Gamma^{\prime 2}}{B_{00}^{2}} u_{0}\right)}{\left(k_{0} a\right)^{2 / 3}}+\ldots \\
& u^{2 / 3} \frac{H_{2 / 3}^{(1)}(u)}{H_{1 / 3}^{(1)}(u)}=-e^{-i \pi / 3} \frac{\Gamma^{\prime 2}}{B_{00}^{2}}+2 e^{-i \pi / 3} \frac{\frac{\Gamma^{\prime}}{B_{00}} u_{0}^{1 / 3} u_{1}\left(1+\frac{\Gamma^{\prime 2}}{B_{00}^{2} u_{0}^{2 / 3}}\right)}{\left(k_{0} a\right)^{2 / 3}}+\ldots, \\
& u^{4 / 3} \frac{H_{2 / 3}^{(1)}(u)}{H_{1 / 3}^{(1)}(u)}=-e^{i \pi / 3} \frac{\Gamma^{\prime}}{B_{00}} u_{0}+\ldots, \\
& u^{7 / 3} \frac{H_{2 / 3}^{(1)}(u)}{H_{1 / 3}^{(1)}(u)}=-e^{i \pi / 3} \frac{\Gamma^{\prime}}{B_{00}} u_{0}^{2}+\ldots, \\
& u^{4 / 3} \frac{H_{2 / 3}^{(1)}{ }^{2}(u)}{H_{1 / 3}^{(1)}(u)}=-e^{-i \pi / 3} \frac{\Gamma^{\prime 2}}{B_{00}^{2}} u_{0}^{2 / 3} \ldots .
\end{aligned}
$$

Substituting all these expansions into (101), and equating to zero the total coefficient of one and the same power of $\left(k_{0} a\right)^{-2 / 3}$ [with an exception of the power $\left(k_{0} a\right)^{2 / 3}$ which leads to $\left.\Gamma^{\prime}=\Gamma^{\prime}\right]$ we obtain relations from which $u_{1}, u_{2}, \ldots$ can be derived in succession. In fact, the $u$ with highest suffix always occurs linearly so that we only have to solve linear equations in order to derive $u_{1}$ from $u_{0}, u_{2}$ from $u_{1}$ and $u_{0}, u_{3}$ from $u_{2}, u_{1}$, and $u_{0}$, etc. The first few coefficients of the expansion (105) for the eigenvalues $u=u_{l}(a)$ can thus be obtained from the relations:

$$
\begin{aligned}
& u_{1}=\frac{a_{00}-\Gamma^{\prime} \frac{a_{01} u_{0}^{2 / 3}}{B_{00}}}{u_{0}^{1 / 3}\left(B_{00}+\frac{\Gamma^{\prime 2}}{B_{00} u_{0}^{2 / 3}}\right)}, \\
& u_{2}=\left(B_{00} u_{0}^{1 / 3}+\frac{\Gamma^{\prime 2}}{R_{\text {กn }} u_{0}^{1 / 3}}\right)^{-1}\left\{\left(-\frac{B_{00} u_{1}^{2}}{6 u_{0}^{2 / 3}}+a_{00} u_{0} u_{1}+a_{10} u_{0}^{2 / 3}\right)-\Gamma^{\prime}\left(\frac{2}{3} \frac{a_{00}}{B_{00}} \frac{u_{1}}{u_{0}^{1 / 3}}\right.\right.
\end{aligned}
$$




$$
\begin{aligned}
& \left.\left.+\frac{a_{11} u_{0}^{4 / 3}}{B_{00}}-u_{1}^{2}\right)+\Gamma^{\prime 2}\left(\frac{u_{1}^{2}}{6 B_{00} u_{0}^{4 / 3}}+\frac{a_{00} u_{1} u_{0}^{1 / 3}}{B_{00}^{2}}-\frac{a_{12}}{B_{00}^{2}}\right)+\frac{\Gamma^{\prime 3} u_{1}^{2}}{B_{00}^{2} u_{0}^{2 / 3}}\right\}, \\
u_{3}= & \left(B_{00} u_{0}^{1 / 3}+\frac{\Gamma^{\prime 2}}{B_{00} u_{0}^{1 / 3}}\right)^{-1}\left[\left\{-B_{00}\left(\frac{u_{1} u_{2}}{3 u_{0}^{2 / 3}}+\frac{u_{1}^{3} u_{0}^{1 / 3}}{3}-\frac{u_{1}^{3}}{27 u_{0}^{5 / 3}}\right)+a_{01} u_{2} u_{0}+\frac{5}{6} a_{01} u_{1}^{2}\right.\right. \\
& \left.+\frac{2}{3} a_{10} \frac{u_{1}}{u_{0}^{1 / 3}}+a_{11} u_{1} u_{0}^{5 / 3}+a_{20} u_{0}^{4 / 3}\right\}+\Gamma^{\prime}\left\{2 u_{1} u_{2}-\frac{u_{1}^{3}}{9 u_{0}}+\frac{e^{-i \pi / 3} a_{21}}{B_{00}}-\frac{a_{01}}{B_{00} u_{0}^{1 / 3}}\left(\frac{2}{3} u_{2}\right.\right. \\
& \left.\left.+u_{1}^{2} u_{0}-\frac{u_{1}^{2}}{9 u_{0}}\right)+2 a_{12} \frac{u_{0}^{1 / 3} u_{1}}{B_{00}}-\frac{4}{3} \frac{a_{11} u_{1} u_{0}^{1 / 3}}{B_{00}}-\frac{a_{22} u_{0}^{2}}{B_{00}}\right\}+\Gamma^{\prime 2}\left\{-\frac{1}{B_{00}}\left(-\frac{u_{1} u_{2}}{3 u_{0}^{4 / 3}}+\frac{4}{3} \frac{u_{1}^{3}}{u_{0}^{1 / 3}}\right.\right. \\
& \left.\left.+\frac{2}{27} \frac{u_{1}^{3}}{u_{0}^{7 / 3}}\right)+\frac{a_{01}}{B_{00}^{2} u_{0}^{2 / 3}}\left(u_{2} u_{0}+\frac{u_{1}^{2}}{2}\right)+\frac{a_{11} u_{1} u_{0}}{B_{00}^{2}}-\frac{a_{23} u_{0}^{2 / 3}}{B_{00}^{2}}\right\}+\Gamma^{\prime 3}\left\{\frac{1}{B_{00}^{2}}\left(\frac{2 u_{1} u_{2}}{u_{0}^{2 / 3}}-\frac{u_{1}^{3}}{3 u_{0}^{5 / 3}}\right)\right. \\
& \left.\left.-\frac{a_{01} u_{1}^{2}}{B_{00}^{3}}+\frac{2 a_{12}}{B_{00}^{3}} \frac{u_{1}}{u_{0}^{1 / 3}}\right\}-\frac{\Gamma^{\prime 4}}{B_{00}^{3}} \frac{u_{1}^{3}}{u_{0}}\right] .
\end{aligned}
$$

\section{Determination of the Eigenvalues Themselves}

We now have to pass from the quantities $u=u_{l}(a)$, determined by (105), to the parameter $l$ which eigenvalue constitutes the complex order of the Legendre function entering in the rigorous mode expansion (24). The connection between $u_{l}(a)$ and $l$ is obtained by combining:

(a) a relation resulting from (9), for $r=a$, and (14), viz:

$$
\frac{l(l+1)}{k_{0}^{2} a^{2}}=C_{l}^{2}=n_{\mathrm{eff}}^{2}(a)-m_{l}^{2}(a)
$$

(b) the expansion (78) for $r=a$, which reads:

$$
\begin{aligned}
m_{l}^{2}(a)=\gamma_{0}^{2}\left\{\frac{u_{l}(a)}{k_{0} a}\right\}^{2 / 3}+2 \gamma_{0} \gamma_{1}\left\{\frac{u_{l}(a)}{k_{0} a}\right\}^{4 / 3}+\left(\gamma_{1}^{2}+2 \gamma_{0} \gamma_{2}\right)\left\{\frac{u_{l}(a)}{k_{0} a}\right\}^{2} & \\
& +2\left(\gamma_{0} \gamma_{3}+\gamma_{1} \gamma_{2}\right)\left\{\frac{u_{l}(a)}{k_{0} a}\right\}^{8 / 3}+\ldots
\end{aligned}
$$

In the latter expansion we substitute for each power of $\left\{u_{\ell}(a) / k_{0} a\right\}^{2 / 3}$ the proper power series following from (105) with the aid of a binomial series. We thus arrive at:

$$
\begin{aligned}
& m_{l}^{2}(a)=\frac{\gamma_{0}^{2} u_{0}^{2 / 3}}{\left(k_{0} a\right)^{2 / 3}}+\frac{2\left(\frac{\gamma_{0}^{2} u_{1}}{3 u_{0}^{1 / 3}}+\gamma_{0} \gamma_{1} u_{0}^{4 / 3}\right)}{\left(k_{0} a\right)^{4 / 3}}+ \frac{\left(\frac{2}{3} \frac{\gamma_{0}^{2} u_{2}}{u_{0}^{1 / 3}}-\frac{\gamma_{0}^{2} u_{1}^{2}}{9 u_{0}^{4 / 3}}+\frac{8}{3} \gamma_{0} \gamma_{1} u_{0}^{1 / 3} u_{1}+\gamma_{1}^{2} u_{0}^{2}+2 \gamma_{0} \gamma_{2} u_{0}^{2}\right)}{\left(k_{0} a\right)^{2}} \\
&+\frac{1}{\left(k_{0} a\right)^{8 / 3}}\left\{\frac{2 \gamma_{0}^{2} u_{3}}{3 u_{0}^{1 / 3}-\frac{2}{9}} \frac{\gamma_{0}^{2} u_{1} u_{2}}{u_{0}^{4 / 3}}+\frac{4}{81} \frac{\gamma_{0}^{2} u_{1}^{3}}{u_{0}^{7 / 3}}+\frac{4}{9} \frac{\gamma_{0} \gamma_{1} u_{1}^{2}}{u_{0}^{2 / 3}}\right. \\
&\left.+\frac{8}{3} \gamma_{0} \gamma_{1} u_{2} u_{0}^{1 / 3}+2\left(\gamma_{1}^{2}+2 \gamma_{0} \gamma_{2}\right) u_{0} u_{1}+2\left(\gamma_{0} \gamma_{3}+\gamma_{1} \gamma_{2}\right) u_{0}^{8 / 3}\right\}+\ldots
\end{aligned}
$$

The quantity $C_{l}^{2}$ is obtained next with the aid of (108), while its square root $C_{l} \sim l /\left(k_{0} a\right)$ can be expanded into powers of $\left(k_{0} a\right)^{-2 / 3}$ by a further application of the binomial power series. The final result starts as follows:

$$
\frac{l}{k_{0} a} \sim C_{l}=n_{\mathrm{eff}}(a)-\frac{\gamma_{0}^{2} u_{0}^{2 / 3}}{2 n_{\mathrm{eff}}(a)\left(k_{0} a\right)^{2 / 3}}-\frac{\gamma_{0}}{\left(k_{0} a\right)^{4 / 3}}\left\{\frac{\frac{\gamma_{0} u_{1}}{3 u_{0}^{1 / 3}+\gamma_{1} u_{0}^{4 / 3}}}{n_{\mathrm{eff}}(a)}+\frac{\gamma_{0}^{3} u_{0}^{4 / 3}}{8 n_{\mathrm{eff}}^{3}(a)}\right\}
$$




$$
\begin{aligned}
& -\frac{1}{\left(k_{0} a\right)^{2}}\left\{\frac{\frac{\gamma_{0}^{2} u_{2}}{3 u_{0}^{1 / 3}}-\frac{\gamma_{0}^{2} u_{1}^{2}}{18 u_{0}^{4 / 3}}+\frac{4}{3} \gamma_{0} \gamma_{1} u_{0}^{1 / 3} u_{1}+\frac{\gamma_{1}^{2} u_{0}^{2}}{2}+\gamma_{0} \gamma_{2} u_{0}^{2}}{n_{\mathrm{eff}}(a)}+\frac{\gamma_{0}^{3}\left(\frac{\gamma_{0} u_{1}}{3} u_{0}^{1 / 3}+\gamma_{1} u_{0}^{2}\right)}{2 n_{\mathrm{eff}}^{3}(a)}+\frac{\gamma_{0}^{6} u_{0}^{2}}{16 n_{\mathrm{eff}}^{5}(a)}\right\} \\
& -\frac{1}{\left(k_{0} a\right)^{8 / 3}}\left\{\frac{\frac{\gamma_{0}^{2} u_{3}}{3 u_{0}^{1 / 3}}-\frac{\gamma_{0}^{2} u_{1} u_{2}}{9 u_{0}^{4 / 3}}+\frac{2}{81} \frac{\gamma_{0}^{2} u_{1}^{3}}{u_{0}^{7 / 3}}+\frac{2}{9} \gamma_{0} \gamma_{1} \frac{u_{1}^{2}}{u_{0}^{2 / 3}}+\frac{4}{3} \gamma_{0} \gamma_{1} u_{2} u_{0}^{1 / 3}+\left(\gamma_{1}^{2}+2 \gamma_{0} \gamma_{2}\right) u_{0} u_{1}+\left(\gamma_{0} \gamma_{3}+\gamma_{1} \gamma_{2}\right) u_{0}^{8 / 3}}{n_{\text {eff }}(a)}\right.
\end{aligned}
$$

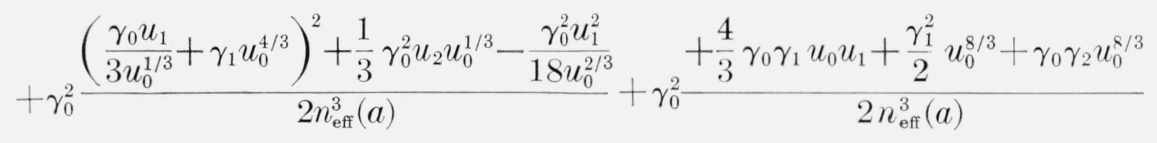

$$
\begin{aligned}
& \left.+\frac{3}{8} \frac{\gamma_{0}^{5}\left(\frac{\gamma_{0} u_{1}}{3} u_{0}+\gamma_{1} u_{0}^{8 / 3}\right)}{n_{\text {eff }}^{5}(a)}+\frac{5}{2048} \frac{\gamma_{0}^{8} u_{0}^{8 / 3}}{n_{\text {eff }}^{7}(a)}\right\}+\ldots
\end{aligned}
$$

The convergence of this expansion can be proved in special cases, one of which will be treated in part III.

According to (40) the exponentials exp (ilt $)$ determine the behavior of the individual modes at large distances. In particular the factor

$$
\left|e^{i l_{0} \theta}\right|=e^{-\operatorname{Im} l_{0} \theta}
$$

is decisive for the attenuation at these distances, if $l_{0}$ represents the eigenvalue with the smallest imaginary part. Obviously, the eigenvalues $l$ depend on the numerical coefficients $\gamma_{j}$ and the quantities $u_{j}$. In view of (107) the latter follow in succession from the other numerical coefficients and from $u_{0}$. In its turn $u_{0}$ has to be determined from (104) which equation, to be discussed now in more detail, represents the zero-order approximation for large values of $\left(k_{0} a\right)^{2 / 3}$.

\section{Equation for the Zero-Order Approximation in the Case of the Electrical Solution}

The equation (104) can be put in the form:

$$
u_{0}^{1 / 3} \frac{H_{2 / 3}^{(1)}\left(u_{0}\right)}{H_{1 / 3}^{(1)}\left(u_{0}\right)}=-\frac{e^{i \pi / 3} \Gamma^{\prime}}{B_{00}} .
$$

The quotient $\Gamma^{\prime} / B_{00}$ can be reduced as follows to the physical data. According to section 6 the parameter $\Gamma^{\prime}=\Gamma /\left(k_{0} a\right)^{2 / 3}$ is given for the electric solution as follows in terms of the atmospheric refractive index $n(a)$, its derivative $n^{\prime}(a)$ and the refractive index $n_{e}$ of the earth:

$$
\Gamma^{\prime}=i\left(k_{0} a\right)^{1 / 3} n(a) \frac{\sqrt{n_{e}^{2}-1}}{n_{e}^{2}}-\frac{a n^{\prime}(a)}{\left(k_{0} a\right)^{2 / 3} n(a)} .
$$

In the second term we can introduce the definition of the effective earth's radius $a_{\text {eff }}$, viz,

Hence, also:

$$
\frac{1}{a_{\mathrm{eff}}}=\frac{1}{a}+\frac{n^{\prime}(a)}{n(a)}
$$

$$
\Gamma^{\prime}=i\left(k_{0} x\right)^{1 / 3} \frac{n(a) \sqrt{n_{e}^{2}-1}}{n_{e}^{2}}-\frac{\left(\frac{a}{a_{\text {eff }}}-1\right)}{\left(k_{0} a\right)^{2 / 3}} .
$$


Further, we find with the aid of (91), (89), and (88):

$$
B_{00}=\left(\frac{3}{2} \Lambda_{1}\right)^{1 / 3}
$$

On the other hand, $\Lambda_{1}$ can be expressed, with the aid of (17), (15), and (6), in terms of higherorder derivatives of the profile function $n(r)$ at the earth's surface. After some reduction we find (again for the electric solution):

$$
\Lambda_{1}=\frac{2 a n^{2}(a)}{a_{\mathrm{eff}}}-\frac{c^{2}}{\omega^{2}}\left\{\left(2+a \frac{d}{d r}\right)\left(\frac{2 n^{\prime 2}}{n^{2}}-\frac{n^{\prime \prime}}{n}\right)\right\}_{r=a},
$$

and a corresponding value for $B_{00}$.

In terms of the new constant:

$$
\delta_{\text {eff }}=\frac{B_{00}}{3^{1 / 3} \Gamma^{\prime}}
$$

which can thus be computed with the aid of (111), (112), and (113), the equation (110) reads:

$$
u_{0}^{1 / 3} \frac{H_{2 / 3}^{(1)}\left(u_{0}\right)}{H_{1 / 3}^{(1)}\left(u_{0}\right)}=-\frac{e^{i \pi / 3}}{3^{1 / 3} \delta_{\text {eff }}} .
$$

Substituting $u_{0}=(-2 \tau)^{3 / 2} / 3$, we get the alternative form:

$$
\sqrt{-2 \tau} \frac{H_{2 / 3}^{(1)}\left\{\frac{(-2 \tau)^{3 / 2}}{3}\right\}}{H_{1 / 3}^{(1)}\left\{\frac{(-2 \tau)^{3 / 2}}{3}\right\}}=-\frac{e^{i \pi / 3}}{\delta_{\text {eff }}},
$$

which is identical with a basic equation in the conventional diffraction theory for a homogeneous atmosphere provided that $\delta$ is replaced there by $\delta_{\text {eff }}$. [See H. Bremmer, Terrestrial Radio Waves, page 43, eq (21a).] The eigenvalues $\tau_{l}$ satisfying this equation are in the first quadrant, and $-2 \tau_{l}$ is defined as a quantity in the $-2^{\text {nd }}$ quadrant.

The techniques for solving (115) in the case of a homogeneous atmosphere can now also be applied here. In particular, the following two expansions could be derived from a Riccati equation equivalent to (115) [loc. cit., page 45, formulas (27) and (28)]:

$$
\begin{aligned}
& \tau_{l}=\tau_{l, 0}-\delta_{\text {eff }}-\frac{2}{3} \tau_{l, 0} \delta_{\text {eff }}^{3}+\frac{1}{2} \delta_{\text {eff }}^{4}-\frac{4}{5} \tau_{l, 0}^{2} \delta_{\text {eff }}^{5}+\ldots, \\
& \tau_{l}=\tau_{l, \infty}-\frac{1}{2 \tau_{l, \infty}} \frac{1}{\delta_{\text {eff }}}-\frac{1}{8 \tau_{l, \infty}^{3}} \frac{1}{\delta_{\text {eff }}^{2}}-\frac{\left(1+\frac{3}{4 \tau_{l, \infty}^{3}}\right)}{12 \tau_{l, \infty}^{2}} \frac{1}{\delta_{\text {eff }}^{3}}-\frac{\left(\frac{7}{3}+\frac{5}{4 \tau_{l, \infty}^{3}}\right)}{32 \tau_{l, \infty}^{4}} \frac{1}{\delta_{\text {eff }}^{4}}+\ldots
\end{aligned}
$$

The numbers $\tau_{l, 0}$ and $\tau_{l, \infty}$ are defined such that $x_{l, 0}=-\frac{1}{3}\left(-2 \tau_{l, 0}\right)^{3 / 2}$ and $x_{l, \infty}=-\frac{1}{3}\left(-2 \tau_{l, \infty}\right)^{3 / 2}$ represent the zeros of $J_{1 / 3}(x)+J_{-1 / 3}(x)$ and of $J_{2 / 3}(x)-J_{-2 / 3}(x)$. We can obtain the corresponding expansions for $(-2 \tau)^{3 / 2}$ and thus for $u_{0}$. The resulting expressions, viz,

$$
\begin{aligned}
u_{0, l} & =\frac{\left(-2 \tau_{l, 0}\right)^{3 / 2}}{3}\left[1-\frac{3}{2} \frac{\delta_{\text {eff }}}{\tau_{l, 0}}+\frac{3}{8} \frac{\delta_{\text {eff }}^{2}}{\tau_{l, 0}^{2}}-\left(1-\frac{1}{16 \tau_{l, 0}^{3}}\right) \delta_{\text {eff }}^{3}\right. \\
& \left.+\left(\frac{5}{4 \tau_{l, 0}}+\frac{3}{128 \tau_{l, 0}^{4}}\right) \delta_{\text {eff }}^{4}-\left(\frac{6}{5} \tau_{l, 0}+\frac{1}{4 \tau_{l, 0}^{2}}-\frac{3}{256 \tau_{l, 0}^{5}}\right) \delta_{\text {eff }}^{5} \ldots\right] \\
u_{0, l} & =\frac{\left(-2 \tau_{l, \infty}\right)^{3 / 2}}{3}\left[1-\frac{3}{4 \tau_{l, \infty}^{2}} \frac{1}{\delta_{\text {eff }}}-\frac{3}{32} \frac{1}{\tau_{l, \infty}^{4}} \frac{1}{\delta_{\text {eff }}^{2}}-\left(\frac{1}{8 \tau_{l, \infty}^{3}}+\frac{5}{128 \tau_{l, \infty}^{6}}\right) \frac{1}{\delta_{\text {eff }}^{3}}-\left(\frac{5}{64} \frac{1}{\tau_{l, \infty}^{5}}+\frac{45}{2048} \frac{1}{\tau^{8}{ }_{l, \infty}}\right) \frac{1}{\delta_{\text {eff }}^{4}}+\ldots\right],
\end{aligned}
$$


are particularly useful for computations concerned with small and large values of $\left|\delta_{\text {eff }}\right|$, respectively.

\section{Complete Evaluation of the Eigenvalues $\mathrm{C}_{l}$}

The final complete procedure for arriving at the numerical values of the parameters $C_{l}$ in the case of a vertical dipole can be summarized as follows. First of all, the physical data to be given are:

(a) the function $n(r)$ representing the smooth profile of the refractive index of the atmosphere,

(b) the refractive index $n_{e}$ of the earth. It is given as follows in terms of the dielectric constant $\epsilon$ of the earth relative to the atmosphere, and the earth's conductivity $\sigma$ in electrostatic units:

$$
n_{e}^{2}=\epsilon+i \frac{4 \pi \sigma}{\omega} .
$$

The complex parameter $\Gamma^{\prime}$ then follows from (111), the effective earth radius being defined by (110a). The parameter $\Lambda_{1}$ can be evaluated next with the aid of (113). It leads to a corresponding value of $B_{00}$ according to (112), whereupon (114) yields the parameter $\delta_{\text {eff }}$. The successive eigenvalues $u_{0, l}$ can be computed next with the aid of (116). This concludes the determination of the zero-order approximation (extended W.K.B. approximation).

The further corrections depend on a sequence of real parameters starting with the quantities $\Lambda_{\jmath}$. With the aid of (17), (15), and (6) we verify that the latter can be represented by:

in which

$$
\Lambda_{j}=a^{j}\left\{\frac{d^{j}}{d r^{j}} M^{2}(r)\right\}_{r=a}-\frac{c^{2}}{\omega^{2}} a^{j-2} \frac{d^{j}}{d r^{j}}\left\{r^{2} n \frac{d^{2}}{d r^{2}}\left(\frac{1}{n}\right)\right\}_{r=a},
$$

$$
M(r)=\frac{r}{a} n(r)
$$

is the modified refractive index of the atmosphere. The second term of (117) is small compared to the first one for most relevant frequencies, but it may become important at the lower end of the spectrum.

With the aid of (83) we derive from $\Lambda_{j}$ the new parameters $\beta_{j}$, next with the aid of (81) from $\beta_{j}$ the quantities $\gamma_{j}$. The quantities $\gamma_{j}$ and $\Lambda_{j}$ now being known, we derive with the aid of (85) the $p_{j}$ 's, whereas the $q_{n j}$ 's follow from (88) if we just know the $\Lambda_{j}$ 's. Having derived both the $q_{n j}$ 's and $p_{j}$ 's, the $t_{n j}$ 's can be computed with the aid of (89). The knowledge of the $t_{n j}$ 's and $q_{n j}$ 's enables the derivation of the $B_{k j}$ 's from (91). Next we compute the $C_{n j}$ 's with the aid of (95). The $a_{n j}$ 's can then be computed according to (102). All numerical coefficients necessary for the derivation in succession of $u_{1}, u_{2}, u_{3} \ldots$ from (107) are then known. Finally, $C_{l}$ can then be computed with the aid of (109), remembering the definition (6) for $n_{\text {eff }}$.

\section{Limiting Cases $\Gamma^{\prime}=0$ and $\Gamma^{\prime}=\infty$}

These special cases are of particular interest. First of all, the evaluation of the final expansion (109) becomes much simpler here than for some finite value of $\Gamma^{\prime}$. On the other hand, the eigenvalues are generally expected to be situated in the complex plane between those corresponding to the two limiting cases.

The value $\Gamma^{\prime}=0$ is approached for a perfectly conducting earth. In fact, the first term of (111) then vanishes since $n_{\theta}=\infty$ while the second term is small but for extreme low frequencies. The other limiting case $\Gamma^{\prime}=\infty$ is characteristic for short waves for which the earth behaves mainly as a dielectric; the factor $n(a)\left(n_{e}{ }^{2}-1\right)^{\frac{1}{2}} / n_{e}{ }^{2}$ in the first term of $\Gamma^{\prime}$ then is of the order of unity so as to have a modulus of this term which is of the order of the very large quantity $\left(k_{0} a\right)^{\frac{1}{3}}$. 
We first deal with the limiting case $\Gamma^{\prime}=0$. The relations $(107)$ representing $u_{1}, u_{2}$. . . in terms of $u_{0}$ here reduce to:

$$
\begin{aligned}
& u_{1}=\frac{a_{00}}{B_{00} u_{0}^{1 / 3}}, \\
& u_{2}=\left(\frac{a_{00}^{2}}{B_{00}^{2}}+\frac{a_{10}}{B_{00}}\right) u_{0}^{1 / 3}-\frac{a_{00}^{2}}{6 B_{00}^{2} u_{0}^{5 / 3}}, \\
& u_{3}=\left(\frac{a_{00}^{2} a_{01}}{B_{00}^{3}}+\frac{a_{11} a_{00}+a_{10} a_{01}}{B_{00}^{2}}+\frac{a_{20}}{B_{00}}\right) u_{0}+\frac{1}{3}\left(\frac{a_{10} a_{00}}{B_{00}^{2}}+2 \frac{a_{00}^{2} a_{01}-a_{00}^{3}}{B_{00}^{3}}\right) \frac{1}{u_{0}}+\frac{5}{54} \frac{a_{00}^{3}}{B_{00}^{3} u_{0}^{3}} .
\end{aligned}
$$

We substitute these expressions into the eigenvalue expansion (109) while approximating $n_{\text {eff }}(a)$ by unity. This approximation amounts to an error which is certainly smaller than that introduced by taking $\Gamma^{\prime}=0$ for some practical conditions. The resulting expansion reads:

$$
\begin{aligned}
\left\{\frac{l}{k_{0} a}\right\}_{\Gamma^{\prime}=0} & =1-\frac{\gamma_{0}^{2} u_{0}^{2 / 3}}{2\left(k_{0} a\right)^{2 / 3}}-\frac{\gamma_{0}}{\left(k_{0} a\right)^{4 / 3}}\left\{\left(\gamma_{1}+\frac{\gamma_{0}^{3}}{8}\right) u_{0}^{4 / 3}+\frac{\gamma_{0}}{3} \frac{a_{00}}{B_{00} u_{0}^{2 / 3}}\right\} \\
& -\frac{1}{\left(k_{0} a\right)^{2}}\left[\left(\frac{\gamma_{1}^{2}}{2}+\gamma_{0} \gamma_{2}+\frac{\gamma_{0}^{3} \gamma_{1}}{2}+\frac{\gamma_{0}^{6}}{16}\right) u_{0}^{2}+\left\{\frac{\gamma_{0}^{2}}{3}\left(\frac{a_{00}^{2}}{B_{00}^{2}}+\frac{a_{10}}{B_{00}}\right)+\frac{4}{3} \frac{\gamma_{0} \gamma_{1} a_{00}}{B_{00}}+\frac{\gamma_{0}^{4} a_{00}}{6 B_{00}}\right\}-\frac{\gamma_{0}^{2} a_{00}^{2}}{9 B_{00}^{2} u_{0}^{2}}\right] \\
& -\frac{1}{\left(k_{0} a\right)^{8 / 3}}\left[\left(\gamma_{0} \gamma_{3}+\gamma_{1} \gamma_{2}+\frac{3}{4} \gamma_{0}^{2} \gamma_{1}^{2}+\frac{\gamma_{0}^{3} \gamma_{2}}{2}+\frac{3}{8} \gamma_{0}^{5} \gamma_{1}+\frac{5}{2048} \gamma_{0}^{8}\right) u_{0}^{8 / 3}\right. \\
& +\left\{\frac{a_{00}^{2} a_{01} \gamma_{0}^{2}}{3 B_{00}^{3}}+\frac{a_{11} a_{00} \gamma_{0}^{2}+a_{10} a_{01} \gamma_{0}^{2}+4 a_{00}^{2} \gamma_{0} \gamma_{1}+\frac{1}{2} \gamma_{0}^{4} a_{00}^{2}}{3 B_{00}^{2}}\right\} \begin{array}{l}
B_{00}^{2 / 3} \\
+
\end{array} \\
& \left.\left.+\frac{\left(\gamma_{1}^{2}+2 \gamma_{0} \gamma_{2}+\gamma_{0}^{3} \gamma_{1}+\frac{\gamma_{0}^{6}}{8}\right) a_{00}+\left(\frac{4}{3} \gamma_{0} \gamma_{1}+\frac{\gamma_{0}^{4}}{6}\right) a_{10}+\frac{a_{20} \gamma_{0}^{2}}{3}}{9 B_{00}^{3}}\right\} \frac{1}{u_{0}^{4 / 3}}+\frac{2}{27} \frac{\gamma_{0}^{2} a_{00}^{3}}{B_{00}^{3}} \frac{1}{u_{0}^{10 / 3}}\right]+\ldots
\end{aligned}
$$

We next pass to the other limiting case $\Gamma^{\prime}=\infty$ which is simpler. Evaluating (107) we first find $u_{1}=0$. However, when determining the limit for $\Gamma^{\prime} \rightarrow \infty$ in the successive terms for the higher order $u_{j}^{\prime}$ 's, we have to take into account the approximation

$$
u_{1} \sim-\frac{a_{01} u_{0}}{\Gamma^{\prime}}
$$

for large $\Gamma^{\prime}$. We then obtain the following expressions:

$$
\begin{aligned}
& u_{1}=0 \\
& u_{2}=-\frac{a_{12}}{B_{00}} u_{0}^{1 / 3} \\
& u_{3}=-\left(\frac{a_{12} a_{01}}{B_{00}^{2}}+\frac{a_{23}}{B_{00}}\right) u_{0} .
\end{aligned}
$$

Substituting these values into (109), again taking $n_{\text {eff }}(a)=1$, the eigenvalue expansion for $\Gamma^{\prime}=\infty$ proves to start with: 


$$
\begin{array}{r}
\left\{\frac{l}{k_{0} a}\right\}_{\Gamma^{\prime}=\infty}=1-\frac{\gamma_{0}^{2} u_{0}^{2 / 3}}{2\left(k_{0} a\right)^{2 / 3}}-\frac{\gamma_{0}}{\left(k_{0} a\right)^{4 / 3}}\left(\frac{\gamma_{0}^{3}}{8}+\gamma_{1}\right) u_{0}^{4 / 3}-\frac{1}{\left(k_{0} a\right)^{2}}\left\{\left(\frac{\gamma_{1}^{2}}{2}+\gamma_{0} \gamma_{2}+\frac{\gamma_{0}^{3} \gamma_{1}}{2}+\frac{\gamma_{0}^{6}}{16}\right) u_{0}^{2}-\frac{\gamma_{0}^{2} a_{12}}{3 B_{00}}\right\} \\
-\frac{1}{\left(k_{0} a\right)^{8 / 3}}\left[\left(\gamma_{0} \gamma_{3}+\gamma_{1} \gamma_{2}+\frac{3}{4} \gamma_{0}^{2} \gamma_{1}^{2}+\frac{\gamma_{0}^{3} \gamma_{2}}{2}+\frac{3}{8} \gamma_{0}^{5} \gamma_{1}+\frac{5}{2048} \gamma_{0}^{8}\right) u_{0}^{8 / 3}\right. \\
\left.-\frac{1}{3}\left\{\gamma_{0}^{2}\left(\frac{a_{12} a_{01}}{B_{00}^{2}}+\frac{a_{23}}{B_{00}}\right)+\frac{4 \gamma_{0} \gamma_{1} a_{12}}{B_{00}}+\frac{\gamma_{0}^{4}}{2} \frac{a_{12}}{B_{00}}\right\} u_{0}^{2 / 3}\right]+\ldots
\end{array}
$$

Examples of the limiting eigenvalue expansions (118) and (119) will be worked out numerically in part III. The first few terms of the expansions (118) and (119) are rather simple even when all its parameters are expressed explicitly in terms of the profile constants $\Lambda_{j}$. The complete evaluation of these parameters results in expansions starting as follows:

$$
\begin{aligned}
&\left\{\frac{l}{k_{0} a}\right\}_{\Gamma^{\prime}=0}=-\frac{\left(\frac{3}{2} \Lambda_{1}\right)^{2 / 3}}{2} \frac{u_{0}{ }^{2 / 3}}{\left(k_{0} a\right)^{2 / 3}}-\frac{\left(\frac{3}{2} \Lambda_{1}\right)^{1 / 3}}{\left(k_{0} a\right)^{4 / 3}}\left[\left\{\frac{3}{16} \Lambda_{1}-\frac{1}{5}\left(1+\frac{\Lambda_{2}}{\Lambda_{1}}\right)\right\} u_{0}^{4 / 3}+\frac{4-\frac{\Lambda_{2}}{\Lambda_{1}}}{30 u_{0}^{2 / 3}}\right] \\
&-\frac{1}{\left(k_{0} a\right)^{2}}\left[\left\{\frac{3}{350}-\frac{3}{20}\left(\Lambda_{1}+\Lambda_{2}\right)+\frac{18}{175} \frac{\Lambda_{2}}{\Lambda_{1}}+\frac{3}{35} \frac{\Lambda_{3}}{\Lambda_{1}}+\frac{9}{64} \Lambda_{1}^{2}-\frac{27}{350} \frac{\Lambda_{2}^{2}}{\Lambda_{1}^{2}}\right\} u_{0}^{2}\right. \\
&\left\{\frac{l}{k_{0} a}\right\}_{\Gamma^{\prime}=\infty}=-\frac{\left(\frac{3}{2} \Lambda_{1}\right)^{2 / 3}}{2} \frac{u_{0}^{2 / 3}}{\left(k_{0} a\right)^{2 / 3}}+\frac{\left.\left(\frac{3}{2}-\frac{\Lambda_{1}}{10}+\frac{\Lambda_{2}}{40}+\frac{16}{105} \frac{\Lambda_{2}}{\Lambda_{1}}+\frac{3}{140} \frac{\Lambda_{3}}{\Lambda_{1}}-\frac{11}{280} \frac{\Lambda_{2}^{2}}{\Lambda_{1}^{2}}\right)-\frac{\left.\left(4-\frac{\Lambda_{2}}{\Lambda_{1}}\right)^{2}\right]}{900 u_{0}^{2}}\right] \ldots}{\left.\left(1+\frac{\Lambda_{2}}{\Lambda_{1}}\right)-\frac{3}{16} \Lambda_{1}\right\} u_{0}^{4 / 3}} \\
&-\frac{1}{\left(k_{0} a\right)^{2}}\left[\left\{\frac{3}{350}-\frac{3}{20}\left(\Lambda_{1}+\Lambda_{2}\right)+\frac{18}{175} \frac{\Lambda_{2}}{\Lambda_{1}}+\frac{3}{35} \frac{\Lambda_{3}}{\Lambda_{1}}+\frac{9}{64} \Lambda_{1}^{2}-\frac{27}{350} \frac{\Lambda_{2}^{2}}{\Lambda_{1}^{2}}\right\} u_{0}^{2}\right. \\
&\left.-\left(\frac{9}{70}+\frac{3}{70} \frac{\Lambda_{2}}{\Lambda_{1}}+\frac{1}{28} \frac{\Lambda_{3}}{\Lambda_{1}}-\frac{9}{280} \frac{\Lambda_{2}^{2}}{\Lambda_{1}^{2}}\right)\right]+\ldots
\end{aligned}
$$

\section{Plane Stratification as a Limiting Case}

The theory for a concentrically symmetric medium can be reduced to that for a medium with a flat stratification (refractive index dependent on an orthogonal coordinate $z$ instead of the radial distance $r$ ). The determination of solutions and eigenvalues of the "flat height-gain differential equation" has been investigated by various authors who discuss extensions of the W.K.B. approximation, comparable to those of the present paper. We mention in particular articles by Cherry [1950], Imai [1956], and Erdelyi [1960]. Recently the problem has been approached in detail by Logan [1961] for the eigenvalues. However, we only considered the spherical problem in view of its direct applicability to far-distance tropospheric propagation. As a matter of fact, results for a flat stratification may be derived from those given here with the aid of a limiting procedure. This will be explained now.

Our basic differential equation has the following form, in view of (8), (9), (15), and the coefficients (17) of the Taylor expansion for $M_{\text {eff }}^{2}(r)$ around $r=a$ :

$$
\left[\frac{d^{2}}{d r^{2}}+k_{0}^{2}\left\{\frac{a^{2}}{r^{2}} \sum_{j=0}^{\infty} \frac{\Lambda_{j}}{a^{j} j !}(r-a)^{j}-\frac{l(l+1)}{k_{0}^{2} r^{2}}\right\}\right]\left(r f_{l}\right)=0 .
$$

We substitute

$$
r=a+z ; \Lambda_{j}=a^{j} \Lambda_{j}^{\prime} ; r f_{l}(r)=F(z) ; l(l+1) \sim l^{2}=\lambda^{2} a^{2},
$$


and next take the limit for $a \rightarrow \infty$. The differential equation resulting reads:

$$
\left\{\frac{d^{2}}{d z^{2}}+\left(k_{0}^{2} \sum_{j=0}^{\infty} \frac{\Lambda_{j}^{\prime}}{j !} z^{j}-\lambda^{2}\right)\right\} F(z)=0 .
$$

On the other hand, the boundary condition (23) becomes, with $\Gamma=\gamma a$,

$$
\frac{\partial F}{\partial z}=\gamma F \text { at } z=0
$$

In view of the relation $\Gamma=\left(k_{0} a\right)^{2 / 3} \Gamma^{\prime}$, and the parameter

$$
B_{00}=\left(\frac{3}{2} t_{10}\right)^{1 / 3}=\left(\frac{3}{2} q_{10}\right)^{1 / 3}=\left(\frac{3}{2} \Lambda_{1}\right)^{1 / 3}=\left(\frac{3}{2} a \Lambda_{1}^{\prime}\right)^{1 / 3},
$$

the equation (104) defining the roots $u_{0}$ may now be represented by:

$$
u_{0}^{1 / 3} \frac{H_{2 / 3}^{(1)}\left(u_{0}\right)}{H_{1 / 3}^{(1)}\left(u_{0}\right)}=-e^{i \pi / 3} \gamma\left(\frac{2}{3 k_{0}^{2} \Lambda_{1}^{\prime}}\right)^{1 / 3} .
$$

The solution of the differential equation (123), with the boundary condition (124) at $z=0$ and the radiation condition at $z=+\infty$, constitutes a very general eigenvalue problem. Its properties can be derived from those in the present paper by substituting (122) into the latter, and then passing to the limit for $a \rightarrow \infty$, As an example we can give the expansions which correspond in the spherical case to (120) and (121):

$$
\begin{aligned}
\left(\frac{\lambda}{k_{0}}\right)_{\gamma=0}= & 1-\frac{\left(\frac{3}{2} \Lambda_{1}^{\prime}\right)^{2 / 3} u_{0}^{2 / 3}}{2 k_{0}^{2 / 3}}-\frac{\left(\frac{3}{2} \Lambda_{1}^{\prime}\right)^{1 / 3}}{k_{0}^{4 / 3}}\left\{\left(\frac{3}{16} \Lambda_{1}^{\prime}-\frac{1}{5} \frac{\Lambda_{2}^{\prime}}{\Lambda_{1}^{\prime}}\right) u_{0}^{4 / 3}-\frac{\Lambda_{2}^{\prime}}{30 \Lambda_{1}^{\prime} u_{0}^{2 / 3}}\right\} \\
& -\frac{1}{k_{0}^{2}}\left[\left(-\frac{3}{20} \Lambda_{2}^{\prime}+\frac{3}{35} \frac{\Lambda_{3}^{\prime}}{\Lambda_{1}^{\prime}}+\frac{9}{64} \Lambda_{1}^{\prime 2}-\frac{27}{350} \frac{\Lambda_{2}^{\prime 2}}{\Lambda_{1}^{\prime 2}}\right) u_{0}^{2}+\left(-\frac{\Lambda_{2}^{\prime}}{40}-\frac{3}{140} \frac{\Lambda_{3}^{\prime}}{\Lambda_{1}^{\prime}}+\frac{11}{280} \frac{\Lambda_{2}^{\prime 2}}{\Lambda_{1}^{\prime 2}}\right)-\frac{\Lambda_{2}^{\prime 2}}{900 \Lambda_{1}^{\prime 2} u_{0}^{2}}\right]+\cdots \\
\left(\frac{\lambda}{k_{0}}\right)_{\gamma=\infty}= & 1-\frac{\left(\frac{3}{2} \Lambda_{1}^{\prime}\right)^{2 / 3} u_{0}^{2 / 3}}{2 k_{0}^{2 / 3}}+\frac{\left(\frac{3}{2} \Lambda_{1}^{\prime}\right)^{1 / 3}}{k_{0}^{4 / 3}}\left(-\frac{3}{16} \Lambda_{1}^{\prime}+\frac{1}{5} \frac{\Lambda_{2}^{\prime}}{\Lambda_{1}^{\prime}}\right) u_{0}^{4 / 3} \\
& -\frac{1}{k_{0}^{2}}\left\{\left(-\frac{3}{20} \Lambda_{2}^{\prime}+\frac{3}{35} \frac{\Lambda_{3}^{\prime}}{\Lambda_{1}^{\prime}}+\frac{9}{64} \Lambda_{1}^{\prime 2}-\frac{27}{350} \frac{\Lambda_{2}^{\prime 2}}{\Lambda_{1}^{\prime 2}}\right) u_{0}^{2}-\left(\frac{1}{28} \frac{\Lambda_{3}^{\prime}}{\Lambda_{1}^{\prime}}-\frac{9}{280} \frac{\Lambda_{2}^{\prime 2}}{\Lambda_{1}^{\prime 2}}\right)\right\}+\cdots
\end{aligned}
$$

The author is most grateful to the Central Radio Propagation Laboratory at Boulder for its assistance in the preparation of this paper. In particular he acknowledges the careful examination of the many formulas by L. Vogler.

\section{References}

Cherry, T. M., Uniform asymptotic formulae for functions with transition points, Trans. Am. Math. Soc. 68, $224-257$ (1950).

Erdélyi, A., Asymptotic solutions of differential equations with transition points or singularities, J. Math. Phys. 1, 16-26 (1960).

Imai, I., A refinement of the WKB method and its application to the electromagnetic wave theory, IRE Trans. on Antennas and Propagation AP-4, No. 3, 233-239 (July 1956).

Langer, R. E., On the connection formulas and the solutions of the wave equation, Phys. Rev. 51, 669-676 (April 1937).

Logan, N. A. (unpublished, 1961).

Pekeris, C. L., Asymptotic solutions for the normal modes in the theory of microwave propagation, J. Appl. Phys. 17, 1108-1124 (Dec. 1946).

(Paper 66D1-172) 